\title{
Directivity of sound radiated from baffled rectangular plates and plate
}

\author{
strips \\ Qi Li ${ }^{1}$, David J Thompson ${ }^{2}$
}

\begin{abstract}
Rectangular plates are important components in structures such as vehicles and bridges. The noise radiated by vibrating plates is mainly determined by three factors: the mean-square vibration, the radiation efficiency of the plates and the directivity of the sound. Although the first two factors have been widely investigated, much less attention has been paid to the directivity. The aim of this study is to investigate the directivity indices for rectangular plates subjected to either a single point force or multiple incoherent forces. Particular attention is given to plates with a large aspect ratio, referred to as plate strips. New definitions of directivity index are introduced that are more appropriate to such plate strips. The vibration of the plates is calculated from a modal superposition method based on approximate modal solutions of the plates with various boundary conditions. The Rayleigh integral method is used to obtain the sound pressure radiated from the vibrating plates, assuming that they are set in an ideal infinite baffle. Directivity indices of sound are firstly determined for plates with a small aspect ratio, and then for plate strips with a larger aspect ratio. Examples are given to illustrate the effects of the structural boundary conditions, and the effect of the baffle. For distributed incoherent excitation, as often found in practice, it is shown that the sound directivity pattern in the far field corresponding to the width direction varies insignificantly along the length of the strips; this is different from a single point force excitation. It is also found that the noise radiation from different concrete plates is approximately omnidirectional in the plane perpendicular to the longitudinal direction. Plates and plate strips are much less
\end{abstract}


directional at high frequencies than the corresponding rigid piston. Nevertheless, the maximum value of directivity increases at high frequency as the number of modes in a onethird octave band increases. The dominant directions of sound radiation from plates are mainly controlled by the acoustical wavelength and vibrational wavelength, while the structural boundary conditions and the presence of the baffle have only a minor influence on them. Finally it is shown that, for the prediction of sound directivity of plate strips under distributed incoherent excitation, a two-dimensional vibro-acoustic model can be used in place of the three-dimensional one.

\section{Keywords}

rectangular plates and strips; sound and vibration; directivity; incoherent excitations; baffle; bridge noise

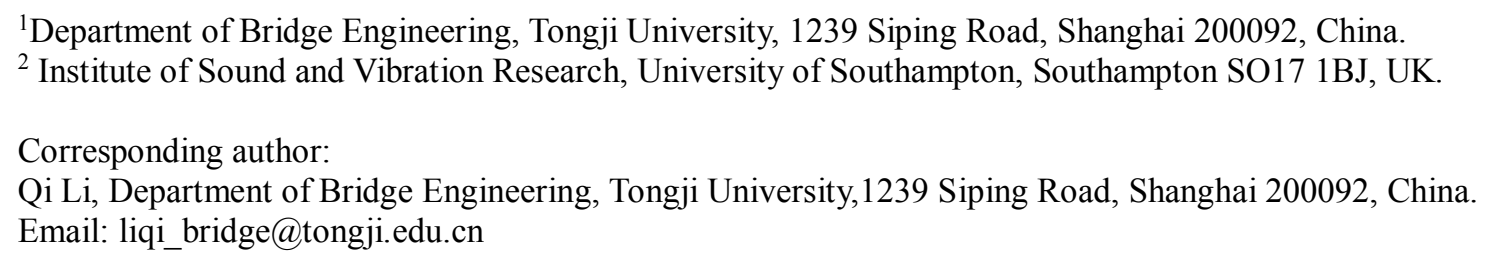

\section{Introduction}

Plates with different sizes and aspect ratios are widely used as important structural components in vehicles, airplanes, industrial machinery, buildings and bridges. They are also important components in the radiation of noise when the structures are subjected to dynamic loading. For the purpose of predicting and mitigating noise from complex structures, it is helpful to understand the sound radiation from vibrating plates; particularly common are those with rectangular shape. Two important factors that influence the radiated sound power of a vibrating plate are the spatially-averaged vibration velocity and the radiation efficiency of the plate. In addition, the sound directivity also plays an important role in determining the distribution of sound pressure for external radiation problems. 
The radiation efficiency of a rectangular plate has been extensively studied, both for individual modes of vibration and for broadband excitation. Wallace [1] derived the modal radiation efficiency of simply-supported rectangular plates set in an infinite rigid baffle, based on the far-field acoustic intensity on a hemisphere of large radius. Gomperts [2, 3] studied the modal radiation efficiency of rectangular plates with different boundary conditions. Berry [4] and Zhang and Li [5] analysed the modal radiation efficiency of a rectangular plate with elastic constraints by using the Rayleigh-Ritz method and Fourier transforms. The effect of plate boundary conditions on the vibration and sound radiation of plates has also been discussed by Park et al. [6], Qiao et al. [7] and Squicciarini et al. [8]. By adopting a modal summation method, Xie et al. [9] investigated the radiation efficiency of a baffled rectangular plate averaged over all possible excitation positions on the plate. The sound radiation of unbaffled plates has also been investigated and compared with the baffled case by Laulagnet [10], Nelisse et al. [11], and Putra and Thompson [12].

Analytical expressions for the sound radiation directivity of simple sound sources such as a dipole, a quadrupole and a baffled circular piston can be found in textbooks, e.g. [13]. However, the sound directivity of vibrating rectangular plates has not been studied as comprehensively as their radiation efficiency. Shuyu Lin [14] used thin plate theory to calculate the vibration of rectangular plates with simple supports on their four edges and then obtained the sound radiation from the plates using the Rayleigh integral method. Hashemi et al. [15] adopted Mindlin plate theory and the Rayleigh integral method to calculate the vibration and sound radiation of moderately thick rectangular plates with various boundary conditions. Both of these studies used three-dimensional plots in terms of spherical coordinates to illustrate the sound directivity pattern for the plates in the far field. The sound directivity expressed in spherical coordinates can be also expressed by $2 \mathrm{D}$ plots with respect to one angular coordinate while the other angular coordinate is fixed [16]. Directivity patterns 
in spherical coordinates may be convenient for plates of small aspect ratio, but they are less suitable for the plate strips of large aspect ratio for which the variations along the longitudinal direction are important.

He et al. [17] developed a method based on Kirchhoff-Love plate theory and the Rayleigh integral to calculate the directivity of the sound radiated by a vibrating stepped circular plate with a free edge. Budarapu et al. [18] also adopted the Rayleigh integral method to investigate the directivity of vibrating plates with attached point masses or springs. Lin and Pan [19] used finite element and boundary element methods to investigate the vibration and sound radiation pattern of a box structure.

Many investigations have been presented of the vibration of plates subjected to a point force or moment excitation, as done by Feit [16]. Tao et al. [20] have investigated the radiation patterns of a thin infinite plate subjected to multiple forces, in phase or out of phase, to account for a machine supported on multiple points on a plate. Michishita et al. [21] have investigated the sound radiation directivity of a plate strip of infinite length with application to steel girder bridges excited by automobiles. However, the vibration of the strip was defined in terms of given waves and not calculated based on specific multiple excitation points. The situation of a finite plate subjected to multiple excitations with moving positions like a bridge excited by moving automobiles or trains has not previously been studied, according to the knowledge of the authors.

The particular motivation for the current work is the external noise radiated from railway bridges. In a recent field test of noise from a concrete U-shaped rail transit bridge, the sound pressure was measured at the same position in the cross-section but at two arbitrary longitudinal positions (N1 and N2, which were $10 \mathrm{~m}$ apart from each other at 1 and $11 \mathrm{~m}$ from the centre of the $30 \mathrm{~m}$ long bridge span, and were both $9.5 \mathrm{~m}$ beneath the centre line of the bridge deck). Fig. 1 shows the sound pressure spectra during the train pass-by time (train 
speed of $80 \mathrm{~km} / \mathrm{h}$ ) at these two positions. It can be seen from the figure that the measured noise levels at the two field points are very similar. This is a practical example suggesting that the angular directivity of sound radiated from plates of a bridge may not change significantly with the longitudinal coordinates (although in the field measurement there are other factors that play a role). In previous studies of this [22-25], the authors have used numerical methods such as the boundary element method to determine sound pressure levels at a range of field points and these have been plotted using contour maps to illustrate the directivity of the sound radiation from specific bridges. Obvious directivity patterns of the bridge noise in a cross-section perpendicular to the bridge axis have been observed but could not be well explained in these studies. Moreover, Li et al. [26] found in a numerical example that a two-dimensional (2D) acoustical model could be used for the prediction of rail and bridge noise as an approximation of the three-dimensional (3D) situation. However, the applicability of this finding requires a more rigorous investigation.

The aim of this study is to investigate the directivity indices for rectangular plates, especially those of large aspect ratio, subjected to either a single point force or multiple incoherent forces. To enhance understanding of the directivity properties of the sound radiated from complex structures, use is made of established and well-recognized theoretical methods to describe the vibration and sound radiation of plates. New definitions are introduced for the directivity of plates that are more applicable to plate strips with a large aspect ratio. To represent the spatial sound field, the sound directivity indices are expressed in cylindrical coordinates because of their applicability to such plate strips. This study focuses on the sound directivity of rectangular plates with various aspect ratios, boundary conditions, and excitation positions. Analytical solutions for the modal frequencies and mode shapes are utilized for a rectangular plate with combinations of clamped, simply-supported or free boundary conditions on each of the four edges. The modal superposition method is then 
applied to calculate the forced vibration of the plate at given positions. The method is verified by comparison with results from a finite element / boundary element model. By assuming, for simplicity, that the plate is set in an ideal infinite baffle, the sound pressure at arbitrary field points is obtained from the Rayleigh integral method [13]. The sensitivity to the baffled approximation is also investigated by comparison with results from the finite element / boundary element model.

\section{Vibration and sound calculation}

The solutions for the natural frequencies and mode shapes derived by Warburton [27] are utilized for a thin rectangular plate with various boundary conditions on each edge. The modal superposition method is used to obtain the forced vibration at any position on the plate. The Rayleigh integral method [13] is then used to calculate the sound pressure at specified field points. This is based on the simplifying assumption that the vibrating plate is mounted in an infinite rigid co-planar baffle.

\subsection{Transverse vibration of a thin plate}

Fig. 2 depicts a thin plate with width $2 a$, length $2 b(b \geq a)$ and thickness $h$. The Cartesian coordinate system is used with coordinates $(x, y)$ and the origin located at the centre of the plate. The mode shapes of the plate can be expressed as the product of characteristic beam functions in the $x$ and $y$ directions

$$
\varphi_{m n}(x, y)=\alpha_{m}(x) \beta_{n}(y)
$$

where $\alpha_{m}(x)$ and $\beta_{n}(y)$ are the characteristic beam functions in the present coordinate system that can be obtained from those given in reference [27]; $\varphi_{m n}(x, y)$ is the mode shape of the plate; $m$ and $n$ are the mode indices for the $x$ and $y$ directions respectively. For the case of simply supported (or sliding) boundaries on two parallel sides, the above equation is exact, but otherwise it is an approximation.

The natural circular frequency of the plate $\omega_{m n}$ corresponding to the mode 
shape $\varphi_{m n}(x, y)$ can be written as

$$
\omega_{m n}=\frac{h \pi^{2}}{2 a^{2}} \sqrt{\frac{E}{12 \rho\left(1-\mu^{2}\right)}\left[G_{x}^{4}+G_{y}^{4}\left(\frac{a}{b}\right)^{4}+2\left(\frac{a}{b}\right)^{2}\left(\mu H_{x} H_{y}-\mu J_{x} J_{y}+J_{x} J_{y}\right)\right]}
$$

where $E, \rho$, and $\mu$ are the Young's modulus, density and Poisson's ratio of the plate; and the coefficients $G, H, J$ are tabulated by Warburton [27] for given boundary conditions and mode indices $(m, n)$ in the $x$ or $y$ directions.

By using the modal superposition method, the vibration velocity amplitude $v(x, y)$ of the plate subjected to a harmonic point force $F \mathrm{e}^{\mathrm{j} \omega t}$ at location $\left(x_{f}, y_{f}\right)$ can be obtained as

$$
v(x, y)=\sum_{m=1}^{M} \sum_{n=1}^{N} \frac{\mathrm{j} \omega F \varphi_{m n}\left(x_{f}, y_{f}\right) \varphi_{m n}(x, y)}{\Gamma_{m n}\left[\omega_{m n}^{2}(1+\mathrm{j} \eta)-\omega^{2}\right]}
$$

where $F$ and $\omega$ are the amplitude and circular frequency of the excitation force; $\mathrm{j}=\sqrt{-1} ; \eta$ is the damping loss factor of the plate; $\Gamma_{m n}$ is the modal mass of the plate; and $M$ and $N$ are the maximum mode indices considered in the $x$ and $y$ directions.

\subsection{Sound radiation from the plate}

In the above equations the vibration of the plate has been expressed in Cartesian coordinates as these are the most logical for a rectangular plate. The sound radiation could be expressed in Cartesian, spherical or cylindrical coordinates. In the current work it is chosen to use cylindrical coordinates as these are more appropriate for plate strips of large aspect ratio.

By using the Rayleigh integral over the plate surface, the sound pressure amplitude at a given field point $(r, \theta, \xi)$ in cylindrical coordinates shown in Fig. 2, can be expressed as [13]

$$
p_{f}(r, \theta, \xi)=\frac{\mathrm{j} \omega \rho_{0}}{2 \pi} \int_{-b}^{b} \int_{-a}^{a} \frac{v(x, y) e^{-\mathrm{j} k r^{\prime}}}{r^{\prime}} \mathrm{d} x \mathrm{~d} y
$$

where $k=\omega / c_{0}$ is the wavenumber of the sound; $c_{0}$ is the speed of sound; $\rho_{0}$ is the density of the air; and $r^{\prime}$ is the distance between the field point and a point $(x, y)$ on the plate surface. The subscript $f$ is introduced to refer to the chosen excitation point used to obtain $v(x, y)$ in Eq. (3). 
In order to characterise the average sound radiation within the length of the plate, the squared pressure is averaged along the longitudinal coordinate $\xi$ as follows

$$
\left|p_{f}(r, \theta)\right|^{2}=\frac{1}{2 b} \int_{-b}^{b}\left|p_{f}(r, \theta, \xi)\right|^{2} \mathrm{~d} \xi
$$

Often a plate is excited by many forces simultaneously or even, in the case of a bridge, by moving forces that cross the plate. The assumption of an array of incoherent forces can be adopted in such situations to simplify the problem. When the plate is excited by $K$ incoherent forces at various positions, the amplitude of the squared sound pressure at a given field point can be expressed as the energy summation of the response to the individual sources

$$
|p(r, \theta, \xi)|^{2}=\sum_{f=1}^{K}\left|p_{f}(r, \theta, \xi)\right|^{2}
$$

Since many vibration modes generally contribute to the sound radiation of a plate for broadband excitation, the sound pressure is first calculated for a fine frequency spacing and then converted to frequency bands. In the present paper, results are presented in conventional one-third octave bands. In each case the sound pressure is calculated at seven discrete frequencies $(0.891,0.926,0.962,1.000,1.039,1.080$, and 1.122 times $f$, where $f$ is centre frequency of interest. The squared sound pressure results are then averaged to give the onethird octave band average. The average only includes half the squared pressure for the first and last frequencies as they are on the boundary of the band.

\subsection{Definition of sound directivity}

Sound directivity indices are usually defined in polar coordinates, especially for circular radiators for which the spatial directivity pattern is axisymmetric. Polar coordinates could still be used to illustrate the sound radiation pattern of a rectangular plate, but they must be depicted in a $3 \mathrm{D}$ diagram. As the current study is focussed particularly on plate strips with a large aspect ratio, the following alternative directivity indices based on cylindrical coordinates are introduced for the sound radiated by the rectangular plate 


$$
\begin{aligned}
& D_{\theta f}(r, \xi)=10 \log _{10} \frac{\left|p_{f}(r, \theta, \xi)\right|^{2}}{\frac{1}{\pi} \int_{-\pi / 2}^{\pi / 2}\left|p_{f}(r, \theta, \xi)\right|^{2} \mathrm{~d} \theta} \\
& D_{\xi f}(r, \theta)=10 \log _{10} \frac{\left|p_{f}(r, \theta, \xi)\right|^{2}}{\frac{1}{2 b} \int_{-b}^{b}\left|p_{f}(r, \theta, \xi)\right|^{2} \mathrm{~d} \xi}
\end{aligned}
$$

The first index shown in Eq. (7), expressed in $\mathrm{dB}$, is referred to here as the angular directivity index, and is used to show the variation of sound intensity in the direction $\theta$ compared with the averaged sound intensity over a circle of radius $r$ at the given longitudinal coordinate $\xi$. The second index defined in Eq. (8), referred to here as the longitudinal directivity index, is used to characterise the variation of sound intensity at the coordinate $\xi$ compared with the averaged sound intensity over a straight line at a distance $r$ from the plate centre and at the given angular coordinate $\theta$. Thus $D_{\theta f}$ is a function of $\theta$ at given values of $(r, \xi)$ whereas $D_{\xi f}$ is a function of $\xi$ at given values of $(r, \theta)$. Larger values of $D_{\theta f}$ or $D_{\xi f}$ indicate that the sound radiation in the given direction is stronger. The directivity indices at various coordinates $(r, \xi)$ or $(r, \theta)$ can be compared with each other. It is noted that the denominators in Eqs. (7)-(8) differ from the conventional ones, which are either the maximum value or the mean value of the squared sound pressure over a specific far-field surface. In this study, they are simply averaged over a line rather than a surface. This approach is used to allow comparisons with a 2D approach. These two indices can be used to represent the spatial sound radiation pattern in both the far field and near field by changing the parameters $r(r>a)$ and $\xi$ or $\theta$.

The directivity indices of the sound radiated by the plate subjected to incoherent forces, i.e. the angular directivity index $D_{\theta}(r, \xi)$ and the longitudinal directivity index $D_{\xi}(r, \theta)$, can be expressed similarly to Eqs (7) and (8) except that $\left|p_{f}\right|^{2}$ is replaced by $|p|^{2}$ from Eq. (6). Moreover, the averaged angular directivity indices $D_{\theta f}(r)$ and $D_{\theta}(r)$ of a plate, excited by a single force and by a set of incoherent forces respectively, are used to represent the average 
sound radiation within the length of the plate, i.e. the average of $D_{\theta f}(r, \xi)$ or $D_{\theta}(r, \xi)$ over $\xi$.

\subsection{Dominant sound radiation direction}

From the angular directivity index $D_{\theta f}(r, \xi)$, the dominant sound radiation angle $\Phi_{f}(r, \xi)$ of a plate can be defined as

$$
\Phi_{f}(r, \xi)=\left.\theta\right|_{D_{\theta f}(r, \xi)=\max \left(D_{\theta f}(r, \xi)\right)}
$$

The above equation can also be applied to the plate excited by incoherent forces when the subscript $f$ is omitted. The variable $\xi$ in Eq. (9) can be also dropped to represent the dominant sound radiation angle corresponding to the averaged angular directivity indices.

The critical frequency $f_{c}$ is the frequency at which $\lambda=\lambda_{b}$ (where $\lambda_{b}$ denotes the wavelength of plate bending waves, and $\lambda$ is the wavelength of the sound in the air), which can be expressed as [13]

$$
f_{\mathrm{c}}=\frac{c_{0}^{2}}{2 \pi h}\left(\frac{12 \rho\left(1-\mu^{2}\right)}{E}\right)^{1 / 2}
$$

In the case of sound radiation from the bending waves of an infinite plate above the critical frequency, $f_{c}$, the sound propagates at the coincidence angle $\Phi_{c}$ to the plate normal [13]

$$
\sin \left(\Phi_{c}\right)=\lambda / \lambda_{b}
$$

The wavelengths $\lambda_{b}$ and $\lambda$ can be expressed as follows [13] :

$$
\begin{gathered}
\lambda_{b}=2 \pi\left(\frac{E h^{2}}{12 \rho\left(1-\mu^{2}\right) \omega^{2}}\right)^{1 / 4} \\
\lambda=\frac{2 \pi}{k}=2 \pi c_{0} / \omega
\end{gathered}
$$

For finite plates subjected to forces within a one-third octave band, the vibration modes with natural frequencies occurring within this band play the dominant role in the vibration of the plate and its sound radiation. The dominant sound radiation angle can be theoretically estimated from Eq. (11) by taking $\lambda_{b}$ as the averaged wavelength for the corresponding mode shapes of the plates in the $x$ direction. Taking a rectangular plate with width $2 a$ and four 
simply-supported edges as an example, the wavelength of the plate for the $m$ th mode is

$$
\lambda_{b}=\frac{4 a}{m}
$$

The dominant sound radiation angle obtained from Eqs. (9) and (11) will be compared in the discussion section.

\section{Results and discussion for plates}

\subsection{Directivity for a rectangular piston}

The directivity of a rectangular piston $(2 a=0.5 \mathrm{~m}, 2 b=0.6 \mathrm{~m})$ is illustrated in this section as a first step. The constant velocity on the plate surface can be obtained from the above formulation by including only the first mode of the plate with four free edges (FFFF edges), $m=1, n=1$.

Fig. 3 shows the angular directivity index $D_{\theta f}(r, \xi)$ of the piston in the far field $(r / a=10)$ for different excitation frequencies $f_{\mathrm{e}}$. These are also expressed in terms of $k a$, where $k$ is the acoustic wavenumber. These results are one-third octave band averages based on seven discrete frequencies, as discussed above. It can be noted from Fig. 3 that the angular directivity index $D_{\theta f}(r, \xi)$ has little dependence on the longitudinal coordinate $\xi$ (see Fig. 2) within the length range of the plate $(-1 \leq \xi / b \leq 1)$. The directivity pattern found can be observed to be similar to that for the circular piston illustrated in [13].

\subsection{Directivity for a rectangular plate subject to a single point force}

Table 1 lists the geometry, material parameters and critical frequencies of the various plates and plate strips used in this study. Plate A, with the parameters listed in the second row of Table 1 , is a typical steel plate with thickness $5 \mathrm{~mm}$ and critical frequency $f_{\mathrm{c}}$ of $2444 \mathrm{~Hz}$. Plate B in Table 1 has twice the thickness of Plate A. Plate C has twice the width of plate A but the same aspect ratio. Plate D has the same thickness and width as Plate A, but a larger aspect ratio of 4 . Plate $\mathrm{E}$ is obtained by doubling all the dimensions of Plate D. Plates F P will be discussed later. 
The excitation force is applied at an arbitrary position on the plate (chosen as $x_{f} / a$ $\left.=-0.37, y_{f} / b=-0.53\right)$ with different one-third octave band centre frequencies. To calculate the vibration of the plate and its sound radiation, a sufficiently large number of modes should be included in the vibration analysis. The maximum values $M$ and $N$ used for each direction are chosen such that the natural frequencies corresponding to the modes $(m=1, n=N)$ and $(m=$ $M, n=1)$ are at least twice the maximum excitation frequency. This leads, for example, to the values $M=13, N=16$ for a frequency of $4000 \mathrm{~Hz}$ for Plate A. The investigation mainly focusses on simply-supported boundary conditions applied along the four edges of the plates (SSSS). Other boundary conditions will be considered later.

Fig. 4 illustrates the angular directivity indices $D_{\theta f}(r, \xi)$ for the plate at the same centre frequencies $\left(f_{\mathrm{e}}=400,2000,4000\right.$ and $\left.10000 \mathrm{~Hz}\right)$ used for the piston. These results correspond to the far field $(r / a=10)$; they are found to be largely independent of $r$ provided that $r / a \geq 5$. The value $r / a=10$ will be used in the remainder of this study. It can be seen that the angular directivity index again has little dependence on the longitudinal coordinate $\xi$. From a comparison with Fig. 3 it is clear that the directivity of the vibrating plate is different from that of a vibrating piston at the same value of $k a$; the piston directivity shows a strong radiation at $0^{\circ}$ to the plate normal, whereas the plate directivity is less strong at $0^{\circ}$ but it contains large lobes at $\pm 49^{\circ}$ and $\pm 30^{\circ}$ respectively at $4000 \mathrm{~Hz}$ and $10000 \mathrm{~Hz}$, as can be seen from Figs. 4(c) and (d). The dominant radiation angles of the plate corresponding to the lobes in Figs 4(c) and (d) match well with those estimated from Eq. (11), which are $51^{\circ}$ and $30^{\circ}$ respectively.

To show the variation with frequency in different cases, and to avoid showing a lot of graphs similar to Fig. 4, Fig. 5(a) shows the maximum values of the averaged angular directivity indices $D_{\theta f}(r)$ for Plates $\mathrm{A}, \mathrm{B}$ and $\mathrm{C}$, which have the same aspect ratio 1.2. Larger values indicate that the radiation is more directional $(0 \mathrm{~dB}$ indicates it is omnidirectional). 
These are plotted against the frequency normalised by the corresponding critical frequency, $f_{\mathrm{e}} / f_{\mathrm{c}}$. Fig. 5(b) displays the associated angle of dominant sound radiation (see Eq. (9)), and Fig. 5(c) gives the number of modes of the plates in each one-third octave band. Figs. 5(d), (e) and (f) illustrate the corresponding results for Plates D and E with a larger aspect ratio of 4 .

The results in Fig. 5(a) indicate that the size of the plate has a strong influence on the maximum value of averaged angular directivity indices, both the normalized excitation frequency $f_{e} / f_{c}$ and wavenumber $k a$ of sound clearly affecting the directivity. This is different from the case of the piston for which the directivity is determined only by the value of $k a$. It can be seen from Fig. 5(a) that the thinner and larger plates generally have larger maximum values of $D_{\theta f}(r)$, i.e. stronger sound directivity in the dominant radiation direction. From Fig. 5(c) it can be seen that the thinner and larger plates also have more vibration modes in a given one-third octave frequency band. Conversely, it can be observed from Figs. 5(d), (e) and (f) that the results for these two plates (D and E) are identical at a given normalised frequency $f_{\mathrm{e}} / f_{\mathrm{c}}$. It can be seen from Fig. $5(\mathrm{~d})$ that a piston of the same size as plate D generally produces larger $D_{\theta f}(r)$ than the plate. This is because the piston is more directional as it has a single vibration mode of infinite wavelength (see Eq. (11)). However, the plates have many bending modes of different wavelengths which radiate sound in different directions.

The dominant radiation angles shown in Fig. 5(b) and Fig. 5(e) vary erratically between 0 and $90^{\circ}$ below the critical frequency. However, in this frequency range the directivity is close to omni-directional as the maximum value of $D_{\theta f}(r)$ is small. For frequencies larger than the critical frequency, the dominant radiation angles can be seen to decrease with increasing frequency. The theoretical result from Eq. (11), also shown in Fig. 5(e), shows a similar trend, which indicates that the theoretical coincidence angle of an infinite plate can be used to estimate the dominant radiation angles of the finite plates above the critical frequency.

For the sound radiated from the plate, unlike the piston, the directivity is not only 
influenced by the acoustic wavelength (and therefore frequency) but also by the bending wavelength of the plate. The acoustic wavelength and averaged bending wavelength of the plate in the frequency band of interest determine the dominant radiation angle (see Eq. (11)), while the number of plate modes in a frequency band is generally related to the maximum value of angular directivity indices. Fig. 6 plots the maximum value of $D_{\theta f}(r)$ against the number of modes in each frequency band for Plates A E. The maximum value of $D_{\theta f}(r)$ generally increases with the number of modes in the frequency band. It can be approximately fitted with the following formula:

$$
D_{\theta f}(r)=4 \log _{10} N
$$

where $N$ is the number of bending modes of the plate in a given one-third octave frequency band, although there is a large spread in the results. The above expression is only valid when $N>2$ as can be seen from Fig. 6 .

\section{Results and discussion for plate strips}

\subsection{Directivity for plate strips under single point force}

The plates used in some engineering applications such as bridge components have large aspect ratios, i.e. $b / a \gg>1$, which can be called 'strips'. In this section, two plate strips (F and G shown in Table 1) with an aspect ratio of 8 are taken as examples to illustrate their sound directivity. They correspond to typical components used in civil engineering structures. Plate F is a $10 \mathrm{~mm}$ thick steel plate with a critical frequency of $1224 \mathrm{~Hz}$, whereas Plate $\mathrm{G}$ is a plate made of concrete with a critical frequency of $70 \mathrm{~Hz}$. The thickness of Plate $\mathrm{G}$ is $250 \mathrm{~mm}$, and it has a bending wavelength of about $1.50 \mathrm{~m}$ at $750 \mathrm{~Hz}$. To use thin plate theory, the thickness of the plate should be no more than $1 / 6$ of the bending wavelength; therefore, it can be considered as a 'thin' plate for frequencies below $750 \mathrm{~Hz}$. Although it is much thicker than the steel plate, this is typical of bridge constructions.

Fig. 7(a) and Fig. 7(b) present the angular directivity indices $D_{\theta f}(r, \xi)$ for $r / a=10$ of the 
steel Plate F subjected to a point force at an arbitrary position on the strip at frequencies below and above the critical frequency: $f_{\mathrm{e}}=1000 \mathrm{~Hz}\left(f_{\mathrm{e}} / f_{\mathrm{c}}=0.82\right)$ and $f_{\mathrm{e}}=2000 \mathrm{~Hz}\left(f_{\mathrm{e}} / f_{\mathrm{c}}=\right.$ 1.64), respectively. Fig. 7(c) and Fig. 7(d) show the results of the concrete Plate G at the frequencies $f_{\mathrm{e}}=50 \mathrm{~Hz}\left(f_{\mathrm{e}} / f_{\mathrm{c}}=0.72\right)$ and $f_{\mathrm{e}}=315 \mathrm{~Hz}\left(f_{\mathrm{e}} / f_{\mathrm{c}}=4.52\right)$, respectively. As can be seen from Figs. 7(b) and (d), the directivity for these frequencies above $f_{c}$ shows obvious dependence on $\xi$ especially for plate F. It may be noted that field points in the region of $r / a=10$ can be very important in practical applications such as for the noise from bridges, ships and vehicles. The dependence of the directivity pattern on the coordinate $\xi$ can produce difficulty in the placement of the microphone around the vibrating structures. However, Figs. 7(a) and (c) indicate the directivity is less dependent on $\xi$ at these lower frequencies (below $f_{c}$ ) where it is also closer to omnidirectional.

In order to quantify the variation of directivity at different frequencies, Fig. 8(a) shows the maximum values of the averaged angular directivity indices $D_{\theta f}(r)$ of Plates $\mathrm{F}$ and $\mathrm{G}$, and Fig. 8(b) displays the associated angles of dominant sound radiation. Fig. 8(a) indicates that the steel plate has higher maximum values in the angular directivity diagrams than the concrete plate. This is at least in part due to differences in the corresponding frequency range. It is also observed from Fig. 8(a) that the piston again produces larger $D_{\theta f}(r)$ than the plate of the same size. The dominant sound radiation angles for Plate F show a similar tendency to those of Plate E (which has the same thickness but different dimensions), as can be seen from a comparison of Fig. 8(b) and Fig. 5(e).

To quantify the variation with position, Fig. 8(c) shows the standard deviation of the maximum values of $D_{\theta f}(r, \xi)$ with respect to the $\xi$ direction, plotted against the normalized frequency. Similarly, Fig. 8(d) presents the standard deviation of the dominant sound radiation angles associated with these maximum values of $D_{\theta f}(r, \xi)$. The standard deviations of the maximum values of $D_{\theta f}(r, \xi)$ are quite small in each frequency band but the standard 
deviation of the dominant sound radiation angles is quite large for the steel plate (Plate F), especially below $f_{c}$. However, the dominant sound radiation angles and their standard deviations are generally very small for the concrete one (Plate G).The directivity indices of plate strips are influenced by many factors such as plate material and size, frequency and position of the excitation forces. Fig. 9(a) compares the maximum values $D_{\theta f}(r)$ of Plates $\mathrm{F}$ and $G$ subjected to a single forces at different positions, and Fig. 9(b) gives the associated dominant radiation angle. It can be seen from these results that the force position has some influence on the sound directivity. It is difficult to obtain a general conclusion for an arbitrary rectangular plate because the directivity is generally irregular and unsymmetrical in the presence of a single excitation force at a particular position on the plate. However, the sound directivity has some interesting and useful properties for strips excited by sets of incoherent forces distributed along the length direction or across the width of the plate. This will be illustrated in the following sections.

\subsection{Directivity for a strip under a set of incoherent forces}

A plate may be excited into vibration by several sets of incoherent forces along lines at various lateral positions or just many forces randomly distributed across the plate. Fig. 10 illustrates the angular directivity indices $D_{\theta}(r, \xi)$ for the same two strips (F and $\mathrm{G}$ ) subjected to incoherent forces across the plate. A total of 25 incoherent forces are equally spaced over the strips in both $x$ and $y$ direction ( 5 by 5 array of forces). The directivity pattern now shows only weak reliance on the coordinate $\xi$. This phenomenon is caused by the averaging of the sound pressure due to the different force positions. Fig. 11 depicts the longitudinal directivity indices $D_{\xi}(r, \theta)$ for Plates $\mathrm{F}$ and $\mathrm{G}$ at three different frequencies at the corresponding dominant radiation angles $\theta$ as shown in the legends of the figures. This is based on the same 5 by 5 array of incoherent forces. It can be seen from Fig. 11 that the sound directivity indices $D_{\xi}(r, \theta)$ generally vary within about $\pm 2 \mathrm{~dB}$ between different $\xi$ locations, apart from those 
near the plate boundaries.

Next, results are presented of the sound radiation of a strip subjected to incoherent forces applied along a line on the strip at a selected lateral position. The structure-borne noise from a railway bridge under a moving train is a particular example of such excitation. Fig. 12 shows the corresponding diagram to Fig. 11 for the same two strips (F and G) but considering a total of 20 incoherent forces that are equally spaced along a line in the length ( $y$ direction) of the strips at a fixed $x$ position $\left(x_{f}=-0.37 a\right)$. It can be observed from a comparison of Fig. 11 and Fig. 12 that the two sets of incoherent forces produce the similar longitudinal directivity indices as well as similar dominant radiation angles (identified in the legend). The above findings match the results given by Li et al. [26] and Song et al. [25] who found that the sound pressure levels of bridge noise vary insignificantly along the moving direction of a train during the passage of the train crossing the bridge.

Fig. 13 presents the equivalent results to Fig. 8 but for the case when 25 incoherent forces are applied across the plates (the same 5 by 5 array of positions). It can be observed from a comparison of the two figures that the incoherent forces produce a more directional radiation than the single force for the steel plate. This is because generally more modes are excited in a given frequency band by the distributed forces; the modes of vibration in this band contribute more significantly to the vibration of the plate compared to other modes of vibration. As a result, the bending wavelength of the plate is more concentrated at a fixed value (see Eq. (11)) and the sound radiation is more directional. The directivity of the concrete plate $\mathrm{G}$ is not obviously affected by the type of excitation because the plate has only a small number of modes of vibration in each frequency band. The dominant radiation angles in Fig. 13(b) remain the same under the two kinds of excitation as they are controlled by the wavelength rather than the number of modes that contribute to the sound radiation. However, the incoherent forces significantly reduce the standard deviations of the maximum angular 
directivity indices and dominant radiation angles (Fig. 13(c,d)) compared with the single force situation (Fig. 8(c,d)). Thus, with distributed excitation the radiation may be more directional but it has a much lower variation along the length of the plate strip.

In order to show the influence of the aspect ratio on the variation of directivity, Fig. 14(a) gives the maximum values of the averaged angular directivity indices $D_{\theta f}(r)$ of Plates $\mathrm{G}, \mathrm{H}$, $\mathrm{I}, \mathrm{J}$ and $\mathrm{K}$ listed in Table 1 . These are all concrete plates of the same width and thickness but different lengths. A total of 25 incoherent forces are applied across these plates ( 5 by 5 array as before). It can be seen from Fig. 14(a) that the aspect ratio b/a has little influence on the maximum values of the averaged angular directivity indices. Fig. 14(b) compares the standard deviation (over $\xi$ ) of the maximum values of $D_{\theta f}(r, \xi)$ for these plates of different aspect ratio. It can be observed that the standard deviations are all less than $1 \mathrm{~dB}$ although they are affected to some extent by the aspect ratio. Results for another set of plates $(\mathrm{L} \sim \mathrm{P}$ in Table 1) are shown in Fig. 15. These plates have a smaller width and thickness than Plate G and again have various aspect ratios, in this case from 4 to 12 .

It can be seen from Fig. 14(a) and Fig. 15(a) that the maximum values of the averaged angular directivity indices generally increase as frequency increases and they are less than 5 $\mathrm{dB}$ for frequencies less than $3 f_{\mathrm{c}}$ and less than $2 \mathrm{~dB}$ for frequencies less than $f_{\mathrm{c}}$. The dominant frequency of noise from concrete railway bridges is usually around $63 \mathrm{~Hz}$ [25] (below $f_{\mathrm{c}}$ ). This means the bridge noise is close to omnidirectional in this frequency range.

Fig. 14(b) and Fig. 15(b) indicate that the standard deviation of the angular directivity over the longitudinal direction is less than $1 \mathrm{~dB}$, which can be ignored for engineering use. The fact that the sound pressure produced by incoherent forces does not depend significantly on the longitudinal location within the plate length is very useful in practice. As a result, the microphones can be installed at one or a small number of arbitrarily chosen positions along the length direction to obtain an estimate of the averaged sound pressure. In other words, the 
$3 \mathrm{D}$ sound directivity pattern for the strip can be approximately represented by a 2D diagram of $D_{\theta}(r)$ in this situation.

Fig. 16 gives the variation of the maximum value of $D_{\theta}(r)$ plotted against the number of modes in each one-third octave band for concrete plates G P subjected to 25 incoherent forces. It can be seen from Fig. 16 that the maximum value of $D_{\theta}(r)$ again generally increases with the number of modes and the fitted formula shown in Eq. (15) can again be applied to these plates for $N>2$.

\subsection{Effect of boundary conditions}

The boundary conditions in practice can be quite complex and will differ from simple supports. To investigate the effect of the boundary conditions, three different combinations are compared in this section in terms of the directivity of sound under incoherent forces (the same 5 by 5 array of positions). These are: four simply-supported edges (SSSS) as above, four clamped edges (CCCC), one clamped edge along the length and three free edges (CFFF) and four free edges (FFFF). Fig. 17(a) presents the maximum values of the averaged sound directivity indices $D_{\theta}(r)$ for Plate $\mathrm{F}$ with these various boundary conditions, and Fig. 17(b) shows the associated dominant radiation angles. Figs. 17(c) and (d) illustrate the corresponding results for Plate G. It can be observed from Fig. 17 that the boundary conditions of the plates have a small influence on the directivity at high frequencies. Compared with Plate G, the boundary conditions of Plate F have less effect on the directivity at high frequencies but a larger influence at low frequencies. This is because the modal density and wavelength of standing waves in the $x$ direction of Plate F are less affected by the boundary conditions at high frequencies but more influenced at low frequencies, as can be seen from Fig. 18. At the lower frequencies, although the maximum directivity indices and dominant angles of the strips are clearly different for various boundary conditions, the sound radiation is close to omnidirectional regardless of the boundary conditions. Fig. 18(b) shows 
that the modal density of Plate G is very low. There is not more than one mode in the width direction in each one-third octave band below $400 \mathrm{~Hz}\left(f_{\mathrm{e}} / f_{\mathrm{c}} \approx 6\right)$.

Fig. 19 gives the standard deviation of the maximum values of $D_{\theta}(r, \xi)$ and of the associated dominant radiation angles with respect to the $\xi$ direction of Plate $\mathrm{F}$ under various boundary conditions. The standard deviations are very small apart from that for the dominant radiation angles at low frequencies where the directivity is in any case quite close to omnidirectional. This again shows that the boundary conditions of the strip have negligible effect on the sound directivity. This is also a very useful finding, indicating that the conclusions from Section 4.2 apply to strips with various different boundary conditions.

\subsection{Comparison between $3 D$ and quasi $2 D$ strips}

In predicting sound radiation, 2D acoustical models have sometimes been used [26] instead of 3D models to reduce the computational effort. The justification for using the 2D models will be illustrated in this section. The sound directivity for strips vibrating in a 3D situation subjected to 20 incoherent forces along a single line is compared with those vibrating with a velocity that is independent of the position along the length direction. A quasi-2D acoustic field can be generated in the latter situation. This can be realized in the above model by adopting free edges at the ends and only including the contribution of vibration from the first mode type $(n=1)$ in the length direction. Fig. 20 compares the averaged angular directivity indices $D_{\theta}(r)$ for the 3D situation of the concrete strips with SSFF and FFFF boundary conditions with those for the corresponding quasi-2D situation. One frequency below and one above the critical frequency are considered in the figure. It can be observed from Fig. 20 that the directivity indices show insignificant differences between the $3 \mathrm{D}$ and quasi-2D results for the dominant radiation directions. If the directivity of a strip with quasi-2D vibration is similar to that of the $3 \mathrm{D}$ result, a $2 \mathrm{D}$ vibro-acoustic model can be used in the approximate prediction of noise radiated from the strips instead of a 3D model 
provided that the radiation powers of the two models are also equivalent. The above findings support the assumption adopted by Li et al. [26].

\subsection{Comparison with numerical results}

A finite element / boundary element model of Plate $G$ has been used to verify the analytical model. For this the indirect boundary element method coded in the software SYSNOISE was used to establish the model. This contains 1936 nodes and 1800 four-node quadrilateral elements. The element size was set to $0.2 \mathrm{~m}$. The finite element method coded in the software ANSYS was adopted to obtain the harmonic vibration of the plate with SSSS edges. The same frequencies were used as in the analytical model. The vibration results of the plate were mapped to the vibrational boundary conditions of the boundary element model. Fig. 21 gives the angular directivity index $D_{\theta}(r, \xi)$ at four selected positions along the length direction of the plate for baffled concrete strips subjected to 5 incoherent forces along a line at $x_{\mathrm{f}=-0.37 a .} D_{\theta}(r, \xi)$ is also shown for the same baffled plate obtained from the analytical method presented in this study. Similar tendencies can be observed from the numerical and analytical results when these two different methods are used for the baffled case. The differences between the results from the two methods are caused by the different approximate discretization used in both methods.

\subsection{Comparison between baffled and unbaffled results}

The condition that the plate is located in an infinite baffle is not necessarily representative of sound radiation from the plates in a complex structure. Another extreme case is a fully unbaffled situation. The results for these two idealised situations are also compared here. Fig. 21 also gives the angular directivity index $D_{\theta}(r, \xi)$ for an unbaffled concrete strip subjected to incoherent forces at the same positions along the length direction of the plate. The unbaffled results shown in Fig. 21 were again obtained from the indirect boundary element method coded in the software SYSNOISE. It can be seen from Fig. 21 that 
the baffle has little influence on the dominant radiation angle of the plate and mainly influences the sound radiation close to the grazing direction. There is still only limited variation of the directivity pattern along the length regardless of the presence of the baffle.

\section{Conclusions}

The directivity of the sound radiation from rectangular plates has been studied in terms of longitudinal and angular directivity indices defined in this study. Analytical solutions have been applied to obtain the vibration of plates and plate strips and their sound radiation. New definitions of directivity have been proposed that may be more appropriate for plate strips of large aspect ratio. Diagrams of these angular and longitudinal directivity indices have then been presented and discussed for typical steel and concrete plates of different aspect ratios and thicknesses with various boundary conditions, and excited by a single force and a set of incoherent forces. The following conclusions can be drawn from the results.

(1) The angular directivity indices of sound from a rectangular plate of small aspect ratio subjected to a single force have little dependence on the longitudinal coordinate $\xi$ provided that the location is within the plate length $(-1 \leq \xi / b \leq 1)$ and in the far field $(r / a \geq 10)$. However, the directivity pattern becomes irregular and unsymmetrical for a plate strip (long plate) subjected to a single force.

(2) The longitudinal directivity pattern of sound from strips shows weak reliance (variation within about $\pm 2 \mathrm{~dB}$ ) on the longitudinal coordinate $\xi(-1<\xi / b<1)$ if a set of incoherent forces is applied on the strips, regardless of the vibrating frequencies considered.

(3) The maximum values of the averaged angular directivity indices of concrete plates excited by incoherent forces are less than $2 \mathrm{~dB}$ below the critical frequency of the plate and are less than $5 \mathrm{~dB}$ at frequencies up to 3 times the critical frequency. In addition, the standard deviations of the maximum angular directivity with the longitudinal direction are less than 1 $\mathrm{dB}$. As a result, both the angular and longitudinal directivity of noise from concrete plates can 
be ignored for engineering use and the noise from concrete bridges can be considered as a line source that is approximately omnidirectional.

(4) Plates and plate strips are much less directional at high frequencies than the corresponding rigid piston. Nevertheless, the maximum value of directivity increases at high frequency as the number of modes in a one-third octave band increases.

(5) The structural boundary conditions of the strips influence the angular directivity pattern at high frequency but not for the dominant range of sound radiation directions, which are associated with the coincidence angle for frequencies above the critical frequency. Moreover, the baffle mainly has a large influence on the sound radiation in directions close to the baffle but not in the dominant sound radiation directions.

(6) The angular directivity pattern of sound from a strip with quasi-2D vibration is similar to that of the 3D result under incoherent excitations in the far field with respect to the width direction. A 2D vibro-acoustic model can therefore be used in the prediction of sound directivity of long strips instead of a 3D model.

All data published in this paper are openly available from the University of Southampton repository at http://dx.doi.org/10.5258/SOTON/D0931.

\section{Acknowledgements}

The study was supported by the Natural Science Foundation of Shanghai (No.15ZR1442800) and National Natural Science Foundation of China (No. 51878501).

\section{References}

[1] Wallace C. Radiation resistance of a rectangular panel. The Journal of the Acoustical Society of America 1972;51(3B):946-52.

[2] Gomperts M. Radiation from rigid baffled, rectangular plates with general boundary conditions. Acta Acustica united with Acustica 1974;30(6):320-7.

[3] Gomperts M. Sound radiation from baffled, thin, rectangular plates. Acta Acustica united with Acustica 1977;37(2):93-102.

[4] Berry A, Guyader JL, Nicolas J. A general formulation for the sound radiation from rectangular, baffled plates with arbitrary boundary conditions. The Journal of the Acoustical Society of America 1990;88(6):2792802.

[5] Zhang X, Li WL. A unified approach for predicting sound radiation from baffled rectangular plates with arbitrary boundary conditions. Journal of Sound and Vibration 2010;329(25):5307-20.

[6] Park J, Mongeau L, Siegmund T. Influence of support properties on the sound radiated from the 
vibrations of rectangular plates. Journal of sound and vibration 2003;264(4):775-94.

[7] Qiao Y, Huang Q, Li L. Influence of boundary conditions on sound radiation characteristic from rectangular plates. Journal of Low Frequency Noise, Vibration and Active Control 2007;26(2):115-33.

[8] Squicciarini G, Thompson D, Corradi R. The effect of different combinations of boundary conditions on the average radiation efficiency of rectangular plates. Journal of Sound and Vibration 2014;333(17):3931-48.

[9] Xie G, Thompson D, Jones C. The radiation efficiency of baffled plates and strips. Journal of Sound and Vibration 2005;280(1):181-209.

[10] Laulagnet B. Sound radiation by a simply supported unbaffled plate. The Journal of the Acoustical Society of America 1998;103(5):2451-62.

[11] Nelisse H, Beslin O, Nicolas J. A generalized approach for the acoustic radiation from a baffled or unbaffled plate with arbitrary boundary conditions, immersed in a light or heavy fluid. Journal of Sound and Vibration 1998;211(2):207-25.

[12] Putra A, Thompson D. Sound radiation from rectangular baffled and unbaffled plates. Applied Acoustics 2010;71(12):1113-25.

[13] Fahy F, Thompson D. Fundamentals of sound and vibration: CRC Press; 2016.

[14] Shuyu L. Study on the radiation acoustic field of rectangular radiators in flexural vibration. Journal of sound and vibration 2002;254(3):469-79.

[15] Hashemi SH, Khorshidi K, Taher HRD. Exact acoustical analysis of vibrating rectangular plates with two opposite edges simply supported via Mindlin plate theory. Journal of Sound and Vibration 2009;322(45):883-900.

[16] Feit D. Pressure Radiated by a Point - Excited Elastic Plate. The Journal of the Acoustical Society of America 1966;40(6):1489-94.

[17] He X, Yan X, Li N. Directivity pattern of the sound radiated from axisymmetric stepped plates. The Journal of the Acoustical Society of America 2016;140(2):1387-96.

[18] Budarapu PR, Narayana T, Rammohan B, Rabczuk T. Directionality of sound radiation from rectangular panels. Applied Acoustics 2015;89:128-40.

[19] Lin TR, Pan J. Sound radiation characteristics of a box-type structure. Journal of Sound and Vibration 2009;325(4):835-51.

[20] Tao J, Liu G, Lam K. Sound radiation of a thin infinite plate in light and heavy fluids subject to multipoint excitation. Applied Acoustics 2001;62(5):573-87.

[21] Michishita K, Sakagami K, Morimoto M, Svensson UP. Sound radiation from an unbaffled elastic plate strip of infinite length. Applied Acoustics 2000;61(1):45-63.

[22] Li Q, Song XD, Wu DJ. A 2.5-dimensional method for the prediction of structure-borne low-frequency noise from concrete rail transit bridges. The Journal of the Acoustical Society of America 2014;135(5):2718-26.

[23] Song $\mathrm{XD}, \mathrm{Li} \mathrm{Q}, \mathrm{Wu} \mathrm{DJ}$. Investigation of rail noise and bridge noise using a combined 3D dynamic model and 2.5 D acoustic model. Applied Acoustics 2016;109:5-17.

[24] Li Q, Thompson DJ. Prediction of rail and bridge noise arising from concrete railway viaducts by using a multilayer rail fastener model and a wavenumber domain method. Proceedings of the Institution of Mechanical Engineers, Part F: Journal of Rail and Rapid Transit 2017:0954409717720839.

[25] Song XD, Wu DJ, Li Q, Botteldooren D. Structure-borne low-frequency noise from multi-span bridges: A prediction method and spatial distribution. Journal of Sound and Vibration 2016;367:114-28.

[26] Li Q, Li WQ, Wu DJ, Song XD. A combined power flow and infinite element approach to the simulation of medium-frequency noise radiated from bridges and rails. Journal of Sound and Vibration 2016;365:134-56.

[27] Warburton G. The vibration of rectangular plates. Proceedings of the Institution of Mechanical Engineers 1954;168(1):371-84. 
Table 1 Geometry, material and critical frequencies of plates and strips used in this study

\begin{tabular}{ccccccccc}
\hline $\begin{array}{c}\text { Plate/strip } \\
\text { identity }\end{array}$ & $\begin{array}{c}\text { Width } \\
2 a \\
(\mathrm{~m})\end{array}$ & $\begin{array}{c}\text { Length } \\
2 b(\mathrm{~m})\end{array}$ & $\begin{array}{c}\text { Aspect } \\
\text { ratio }\end{array}$ & $\begin{array}{c}\text { Thickness } \\
h(\mathrm{~mm})\end{array}$ & $\begin{array}{c}\text { Density } \\
\rho \\
\left(\mathrm{kg} / \mathrm{m}^{3}\right)\end{array}$ & $\begin{array}{c}\text { Damping } \\
\text { loss } \\
\text { factor }\end{array}$ & $\begin{array}{c}\text { Elastic } \\
\text { modulus } \\
E(\mathrm{GPa})\end{array}$ & $\begin{array}{c}\text { Critical } \\
\text { frequency } \\
f_{\mathrm{c}}(\mathrm{Hz})\end{array}$ \\
\hline $\mathrm{A}$ & 0.5 & 0.6 & 1.2 & 5 & 7800 & 0.01 & 200 & 2444 \\
$\mathrm{~B}$ & 0.5 & 0.6 & 1.2 & 10 & 7800 & 0.01 & 200 & 1222 \\
$\mathrm{C}$ & 1.0 & 1.2 & 1.2 & 5 & 7800 & 0.01 & 200 & 2444 \\
$\mathrm{D}$ & 0.5 & 2.0 & 4.0 & 5 & 7800 & 0.01 & 200 & 2444 \\
$\mathrm{E}$ & 1.0 & 4.0 & 4.0 & 10 & 7800 & 0.01 & 200 & 1222 \\
$\mathrm{~F}$ & 1.0 & 8.0 & 8.0 & 10 & 7800 & 0.01 & 200 & 1222 \\
$\mathrm{G}$ & 3.0 & 24.0 & 8.0 & 250 & 2600 & 0.01 & 35 & 70 \\
$\mathrm{H}$ & 3.0 & 18.0 & 6.0 & 250 & 2600 & 0.04 & 35 & 70 \\
$\mathrm{I}$ & 3.0 & 12.0 & 4.0 & 250 & 2600 & 0.04 & 35 & 70 \\
$\mathrm{~J}$ & 3.0 & 6.0 & 2.0 & 250 & 2600 & 0.04 & 35 & 70 \\
$\mathrm{~K}$ & 3.0 & 3.0 & 1.0 & 250 & 2600 & 0.04 & 35 & 70 \\
$\mathrm{~L}$ & 2.0 & 24.0 & 12.0 & 125 & 2600 & 0.04 & 35 & 140 \\
$\mathrm{M}$ & 2.0 & 20.0 & 10.0 & 125 & 2600 & 0.04 & 35 & 140 \\
$\mathrm{~N}$ & 2.0 & 16.0 & 8.0 & 125 & 2600 & 0.04 & 35 & 140 \\
$\mathrm{O}$ & 2.0 & 12.0 & 6.0 & 125 & 2600 & 0.04 & 35 & 140 \\
$\mathrm{P}$ & 2.0 & 8.0 & 4.0 & 125 & 2600 & 0.04 & 35 & 140 \\
\hline
\end{tabular}

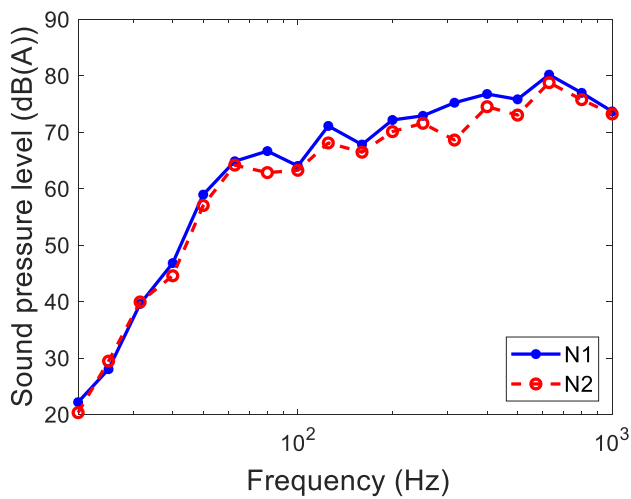

Figure 1. Spectra of sound pressure levels during the train pass-by time $(80 \mathrm{~km} / \mathrm{h})$ at the same plane position but two different longitudinal positions along a real U-shaped girder bridge 


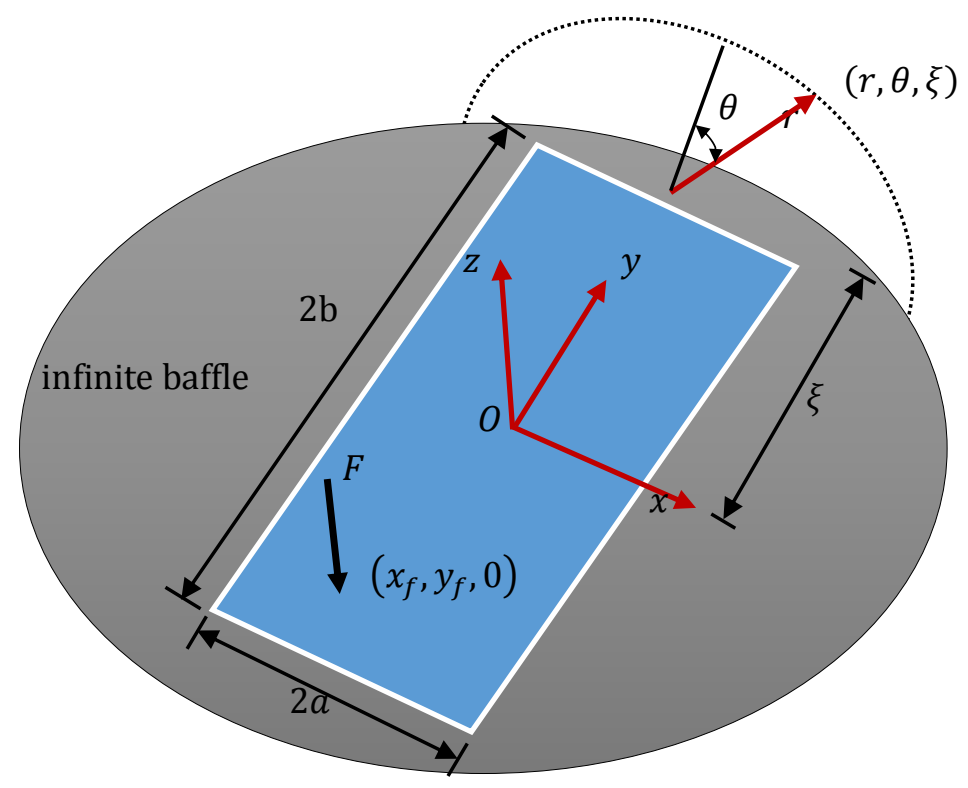

Figure 2. A baffled rectangular plate associated with the main geometrical parameters and defined coordinate systems

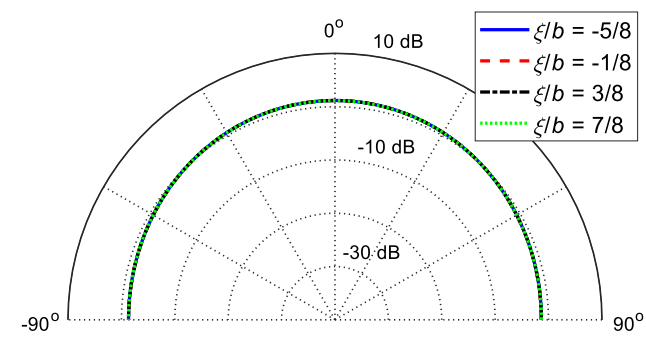

(a)

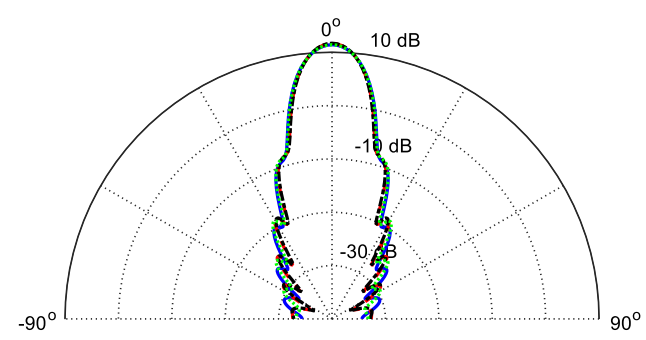

(c)

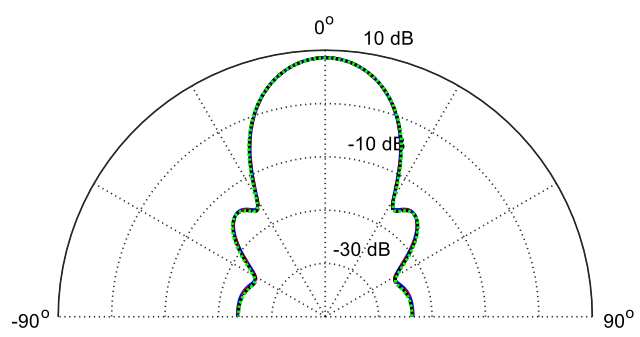

(b)

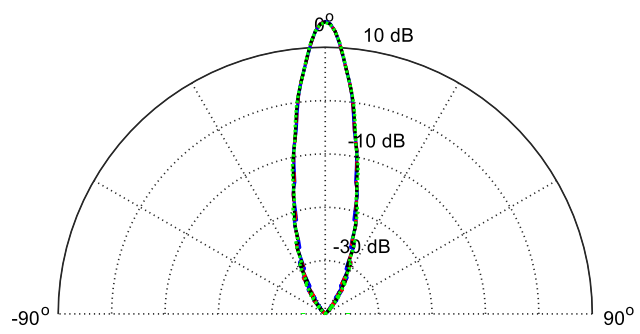

(d)

Figure 3. Angular directivity indices $D_{\theta f}(r, \xi)$ for a rectangular piston $(r / a=10)$ : (a) $f_{\mathrm{e}}=400 \mathrm{~Hz}, k a=$ 1.83 ; (b) $f_{\mathrm{e}}=2000 \mathrm{~Hz}, k a=9.16$; (c) $f_{\mathrm{e}}=4000 \mathrm{~Hz}, k a=18.3$; (d) $f_{\mathrm{e}}=10000 \mathrm{~Hz}, k a=45.8$ 


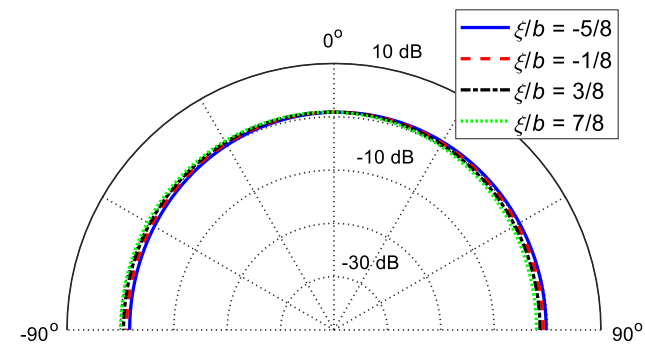

(a)

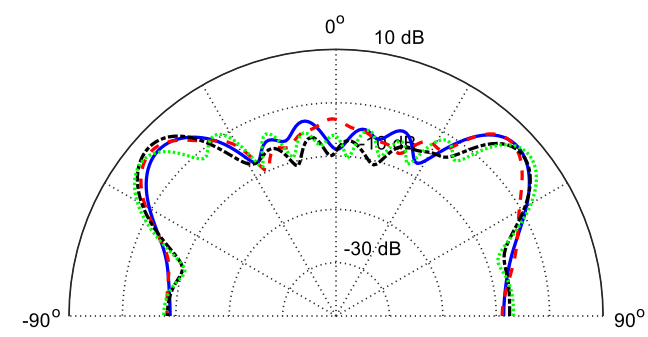

(c)

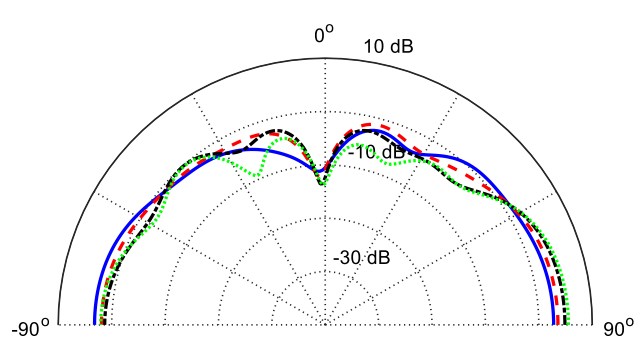

(b)

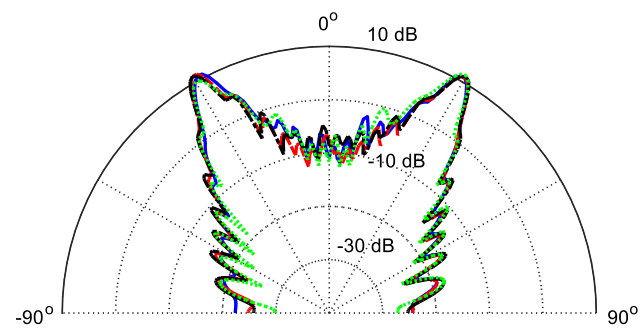

(d)

Figure 4. Angular directivity indices $D_{\theta f}(r, \xi)$ for a plate with simple boundary conditions along the four edges (SSSS) and under a single force (Plate A, $f_{\mathrm{c}}=2444 \mathrm{~Hz}, x_{f} / a=-0.37, y_{f} / b=-0.53$ ): (a) $f_{\mathrm{e}}=400 \mathrm{~Hz}, f_{\mathrm{e}}$ $/ f_{\mathrm{c}}=0.16, k a=1.83$; (b) $f_{\mathrm{e}}=2000 \mathrm{~Hz}, f_{\mathrm{e}} / f_{\mathrm{c}}=0.82, k a=9.16$; (c) $f_{\mathrm{e}}=4000 \mathrm{~Hz}, f_{\mathrm{e}} / f_{\mathrm{c}}=1.64, k a=18.3$; (d) $f_{\mathrm{e}}$ $=10000 \mathrm{~Hz}, f_{\mathrm{e}} / f_{\mathrm{c}}=4.10, \mathrm{ka}=45.8$ 


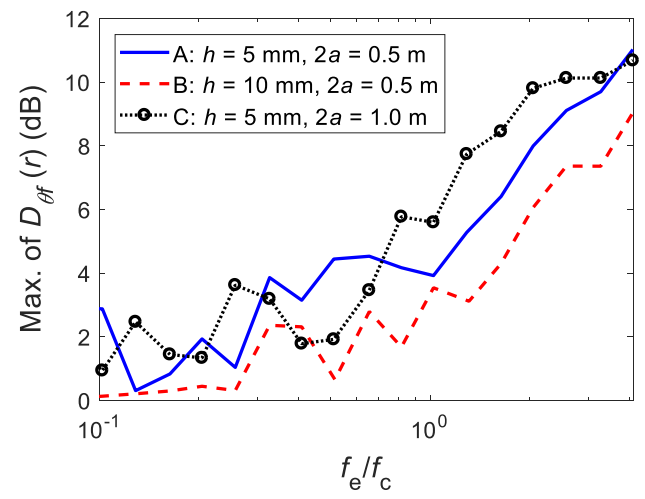

(a)

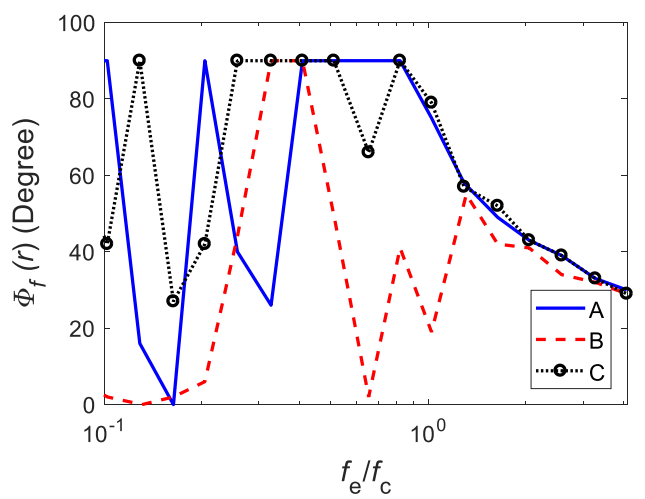

(b)

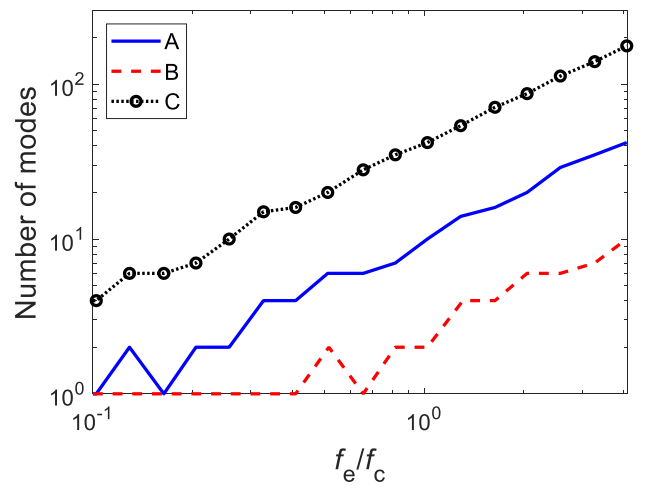

(c)

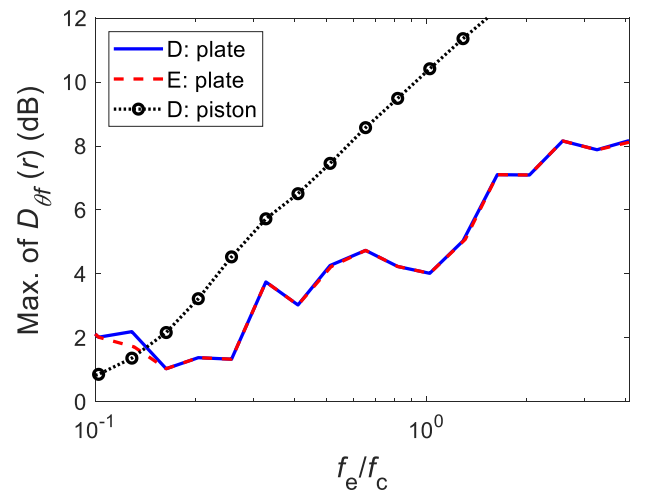

(d)

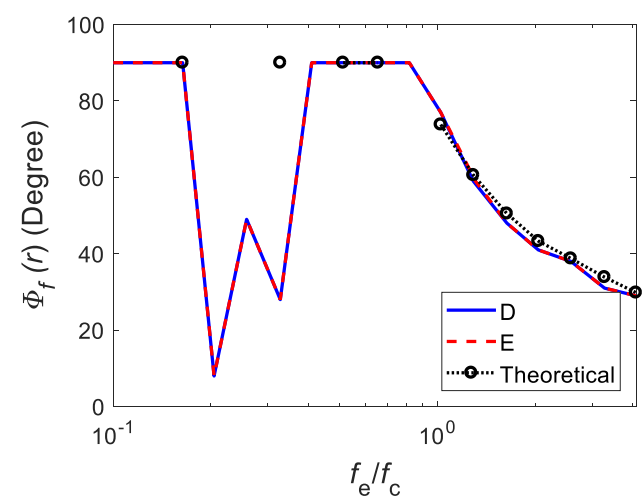

(e)

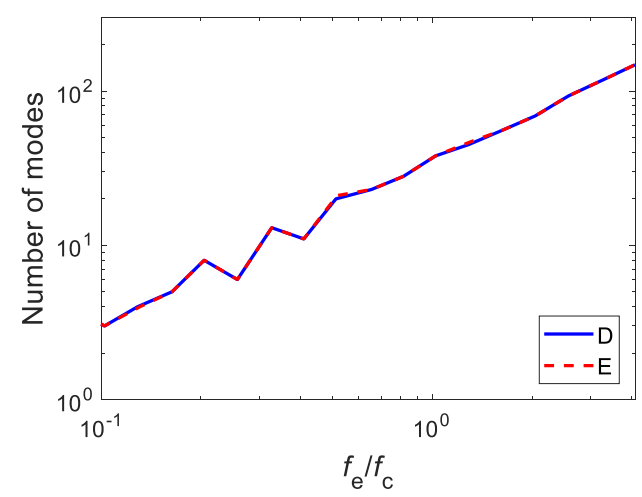

(f)

Figure 5. Variation of averaged angular directivity and modal density with frequency for different plates (SSSS, $x_{f} / a=-0.37, y_{f} / b=-0.53, r / a=10$ ): (a) maximum value of $D_{\theta f}(r)$, aspect ratio 1.2 ; (b) dominant radiation angle $\theta_{\mathrm{m}}$, aspect ratio 1.2 ; (c) number of modes in each one-third octave band, aspect ratio 1.2;

(d) maximum value of $D_{\theta f}(r)$, aspect ratio 4; (e) dominant radiation angle $\theta_{\mathrm{m}}$, aspect ratio 4.0; (f) number of modes in each one-third octave band, aspect ratio 4.0 


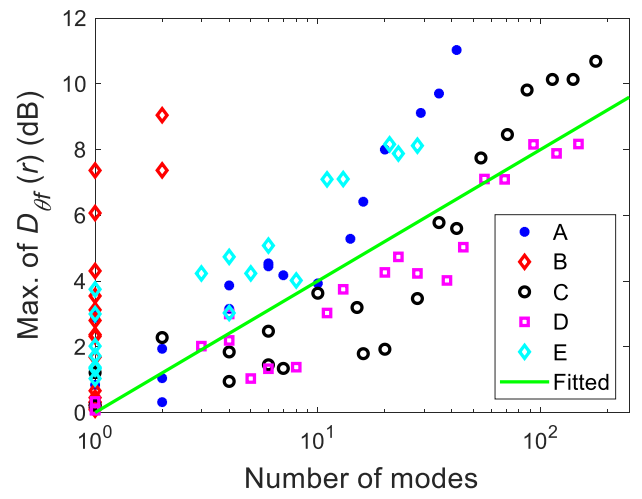

Figure 6. Maximum value of $D_{\theta f}(r)$ against number of modes in each one-third octave band for steel plates of different sizes and subjected to single force

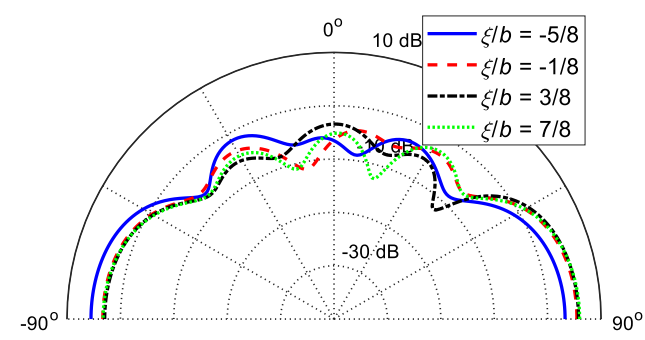

(a)

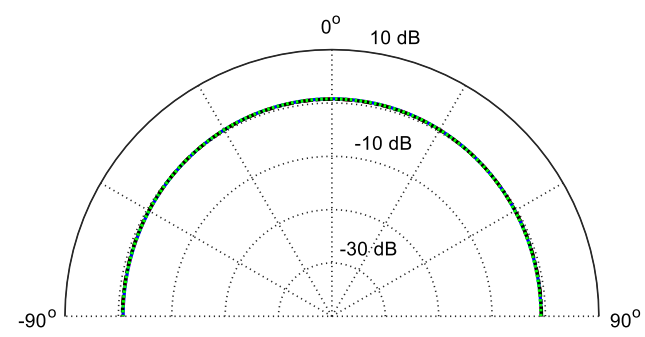

(c)

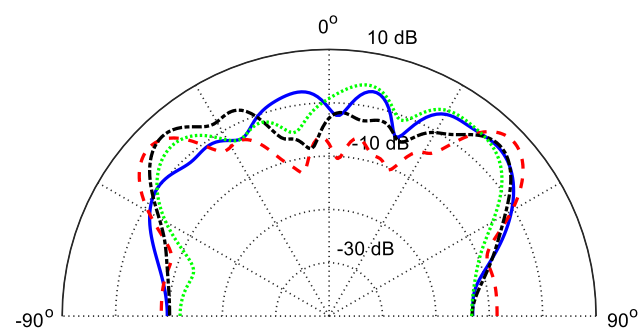

(b)

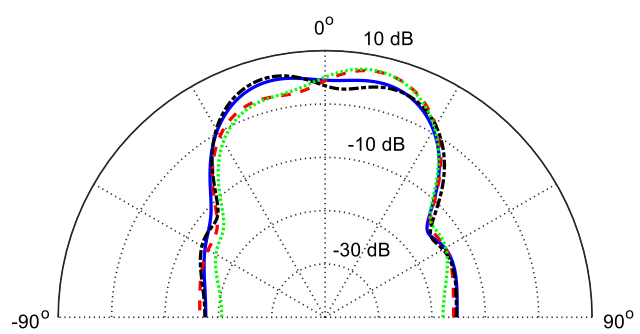

(d)

Figure 7. Angular directivity indices $D_{\theta f}(r, \xi)$ for plate strips (SSSS, $x_{f} / a=-0.37, y_{f} / b=-0.51, r / a=10$ ): (a) Plate F, $f_{\mathrm{e}}=1000 \mathrm{~Hz}, f_{\mathrm{e}} / f_{\mathrm{c}}=0.82, k a=9.16$; (b) Plate F, $f_{\mathrm{e}}=2000 \mathrm{~Hz}, f_{\mathrm{e}} / f_{\mathrm{c}}=1.64, k a=18.3$; (c) Plate $\mathrm{G}, f_{\mathrm{e}}=50 \mathrm{~Hz}, f_{\mathrm{e}} / f_{\mathrm{c}}=0.72, k a=1.34 ;$ (d) Plate $\mathrm{G}, f_{\mathrm{e}}=315 \mathrm{~Hz}, f_{\mathrm{e}} / f_{\mathrm{c}}=4.52, k a=8.66$ 


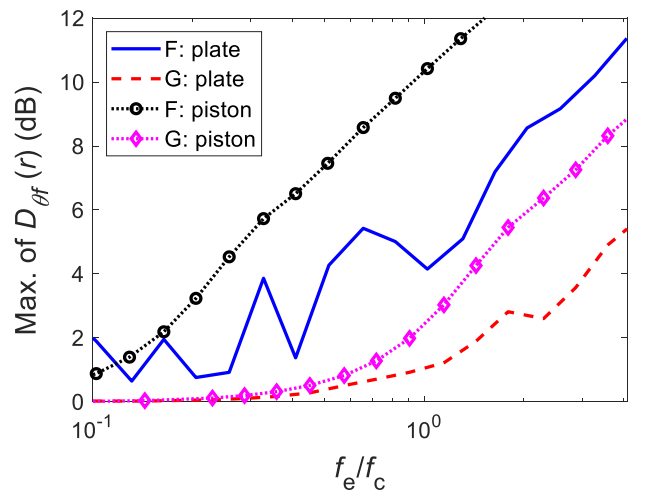

(a)

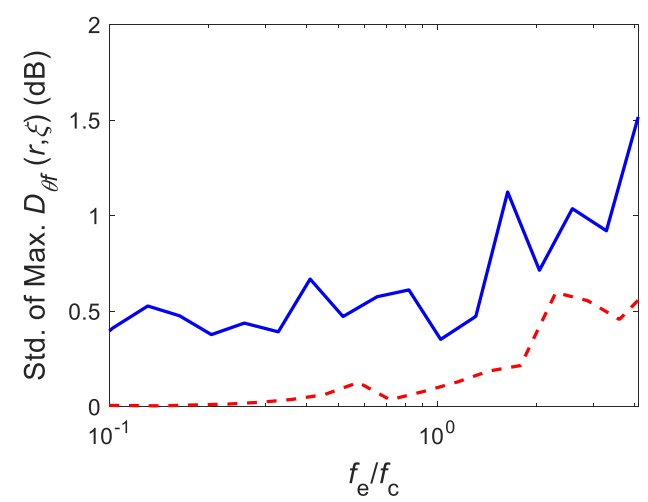

(c)

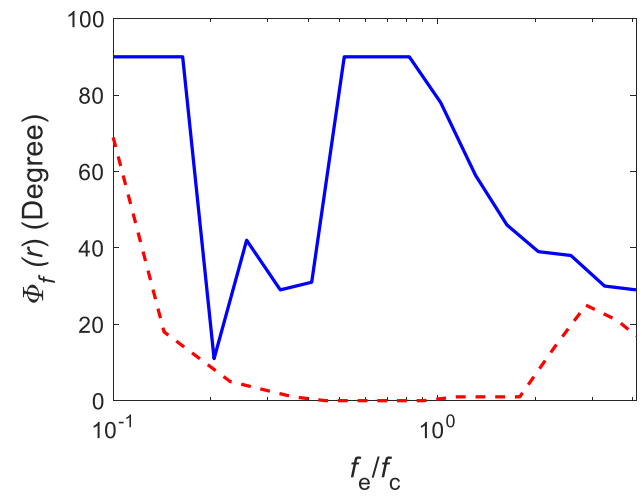

(b)

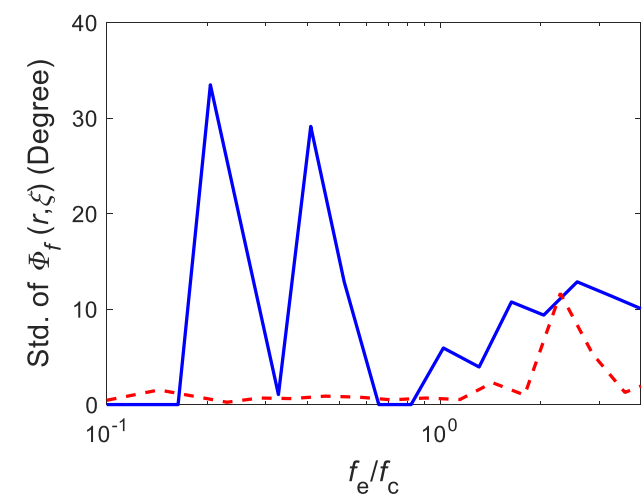

(d)

Figure 8. Variation of averaged angular directivity with frequency (SSSS, $x_{f} / a=-0.37, y_{f} / b=-0.53, r / a=$ 10): (a) maximum value of $D_{\theta f}(r)$; (b) dominant radiation angle; (c) standard deviation of maximum values of $D_{\theta f}(r, \xi)$ with respect to $\xi$ direction; (d) standard deviation of the dominant sound radiation angles with respect to $\xi$ direction

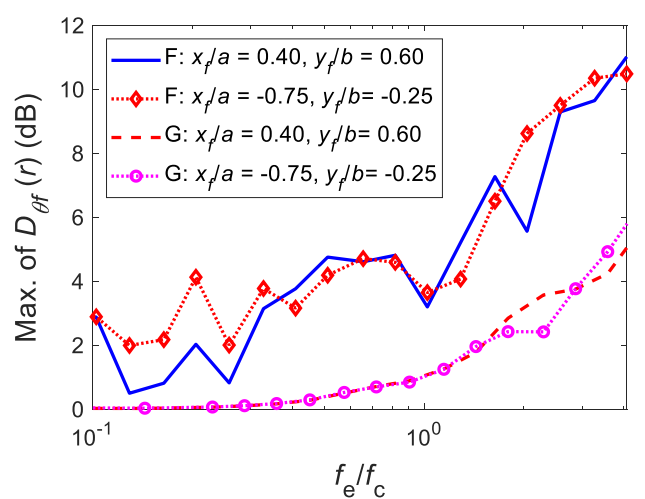

(a)

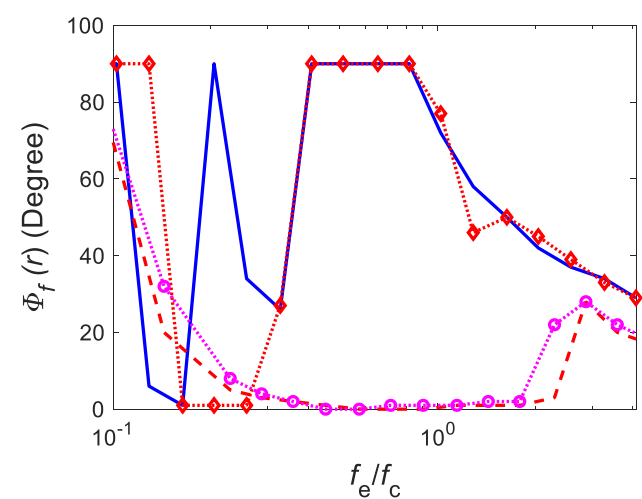

(b)

Figure 9. Variation of averaged angular directivity with frequency subjected to a single force at different positions (SSSS, $r / a=10$ ): (a) maximum value of $D_{\theta f}(r)$; (b) dominant radiation angle 


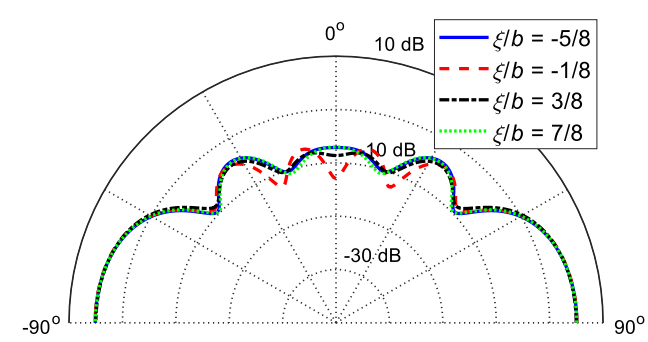

(a)

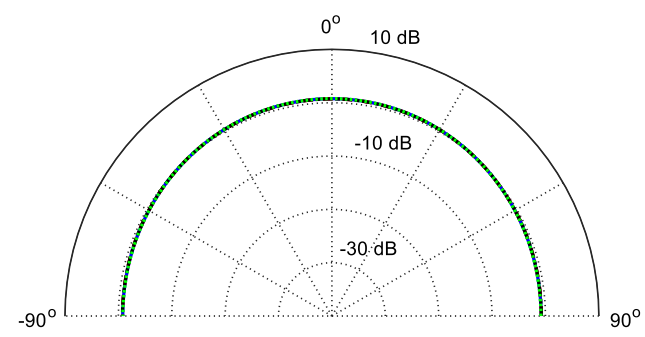

(c)

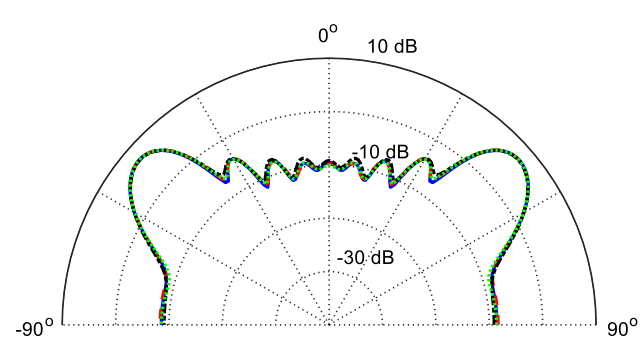

(b)

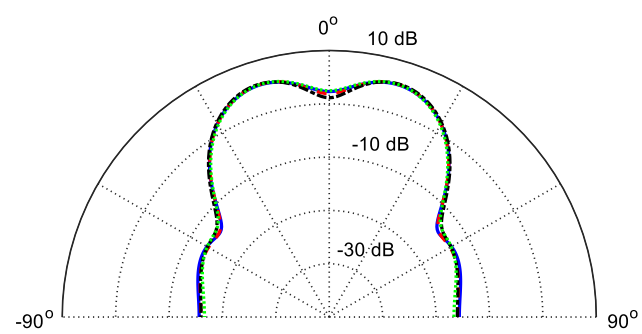

(d)

Figure 10. Angular directivity indices $D_{\theta}(r, \xi)$ for plate strips subjected to 25 incoherent forces across the plate (SSSS, $r / a=10$ ): (a) Plate F, $f_{\mathrm{e}}=1000 \mathrm{~Hz}, f_{\mathrm{e}} / f_{\mathrm{c}}=0.82, k a=9.16$; (b) Plate F, $f_{\mathrm{e}}=2000 \mathrm{~Hz}, f_{\mathrm{e}} / f_{\mathrm{c}}=$ $1.64, k a=18.3$; (c) Plate G, $f_{\mathrm{e}}=50 \mathrm{~Hz}, f_{\mathrm{e}} / f_{\mathrm{c}}=0.72, k a=1.34$; (d) Plate G, $f_{\mathrm{e}}=315 \mathrm{~Hz}, f_{\mathrm{e}} / f_{\mathrm{c}}=4.52, k a=$ 8.66

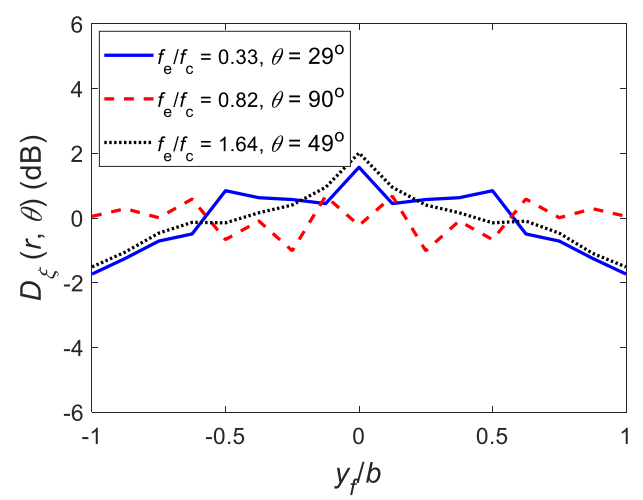

(a)

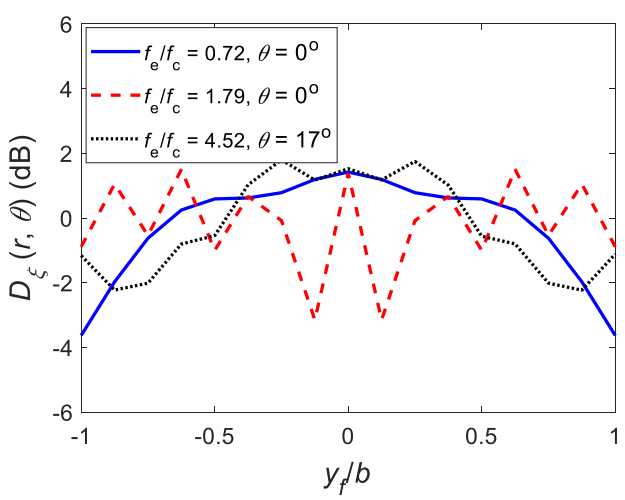

(b)

Figure 11. Longitudinal directivity indices $D_{\xi}(r, \theta)$ at dominant radiation angles for the plate strips subjected to 25 incoherent forces across the plate (SSSS, $r / a=10$ ): (a) Plate F; (b) Plate G

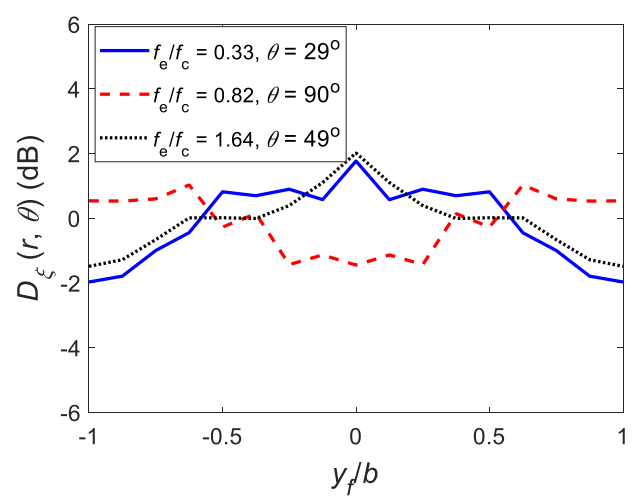

(a)

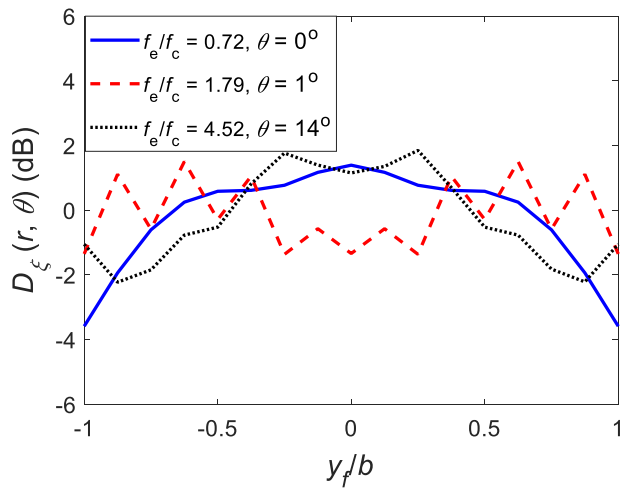

(b)

Figure 12. Longitudinal directivity indices $D_{\xi}(r, \theta)$ at dominant radiation angles for the plate strips subjected to 20 incoherent forces along the length direction (SSSS, $x_{f} / a=-0.37, r / a=10$ ): (a) Plate F; (b)

Plate G 


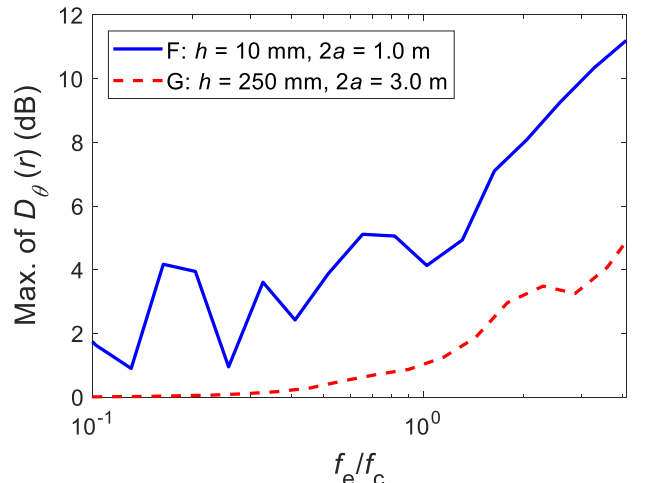

(a)

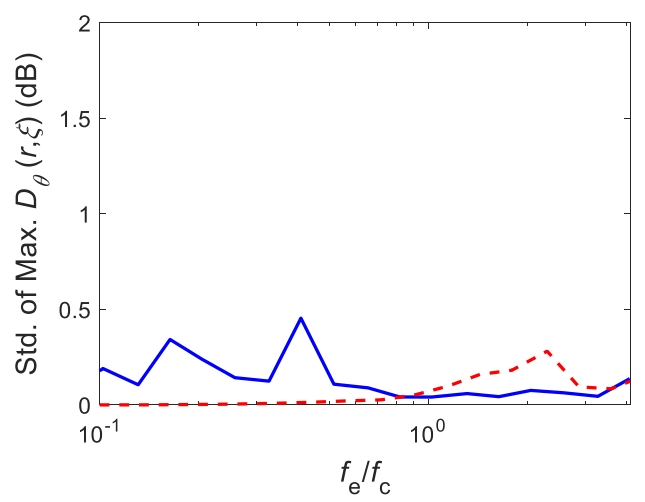

(c)

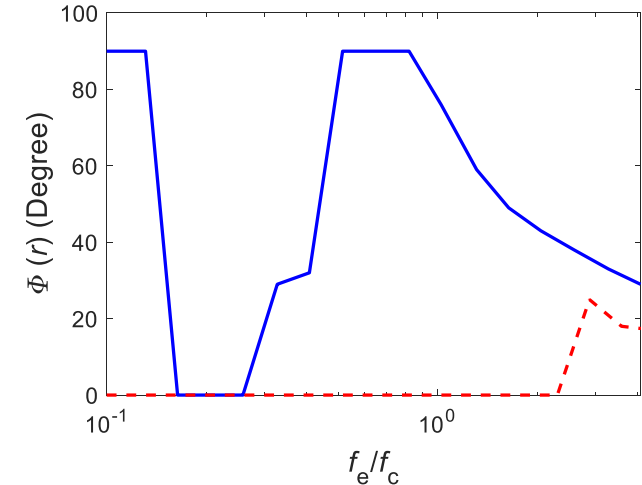

(b)

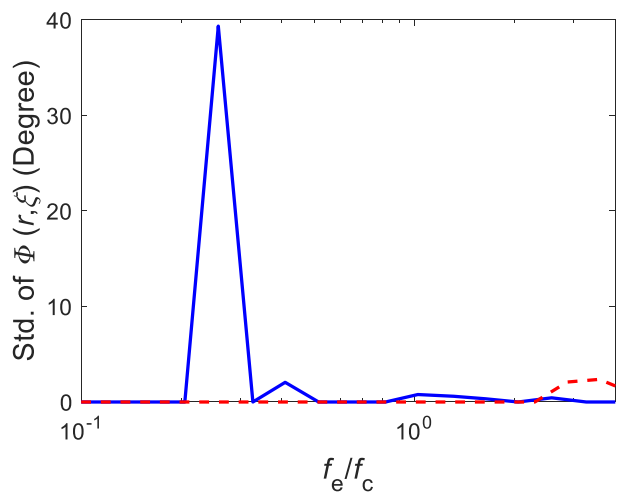

(d)

Figure 13. Variation of averaged angular directivity with frequency for the plate strips subjected to 25 incoherent forces across the plate (SSSS, $r / a=10$ ): (a) maximum value of $D_{\theta f}(r)$; (b) dominant radiation angle; (c) standard deviation of maximum values of $D_{\theta f}(r, \xi)$ with respect to $\xi$ direction; (d) standard deviation of the dominant sound radiation angles with respect to $\xi$ direction

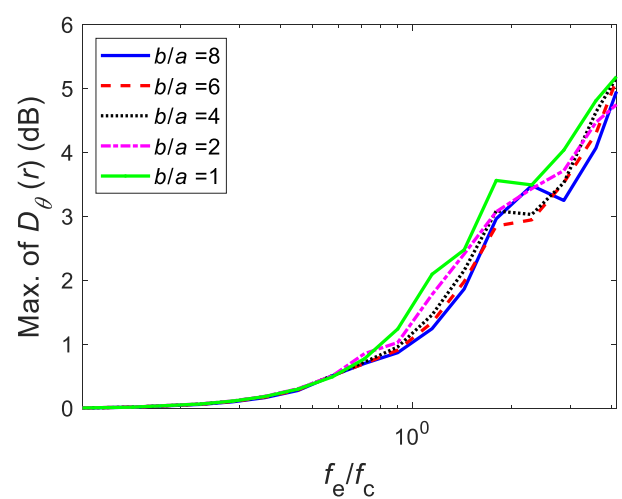

(a)

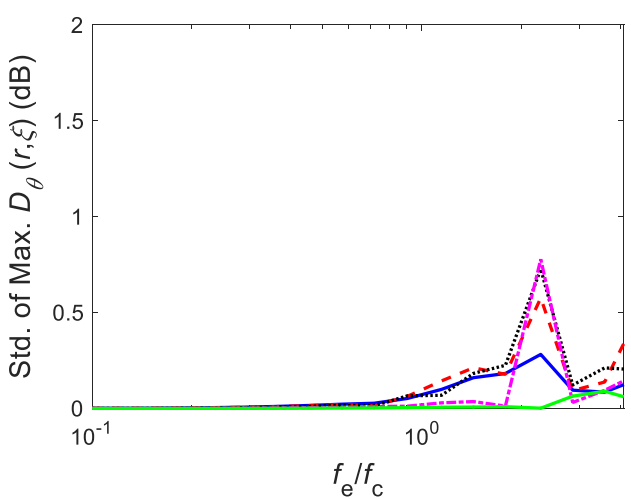

(b)

Figure 14. Variation of averaged angular directivity with frequency for the concrete plate strips of different aspect ratio (b/a) and subjected to 25 incoherent forces across the plate (SSSS, $r / a=10, h=250 \mathrm{~mm}$ ): (a) maximum value of $D_{\theta}(r)$; (b) standard deviation of maximum values of $D_{\theta}(r, \xi)$ with respect to $\xi$ direction 


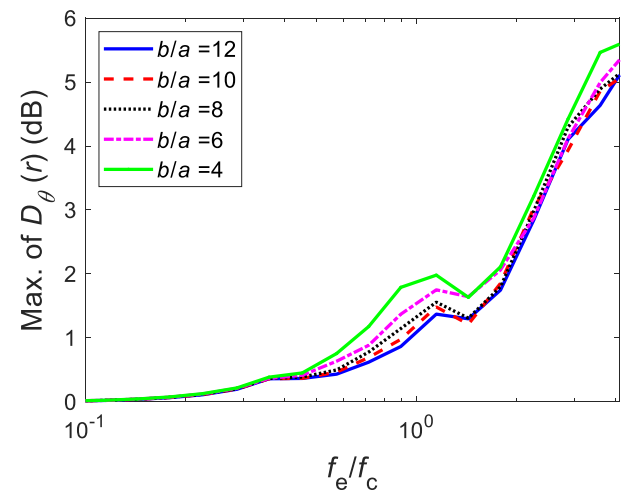

(a)

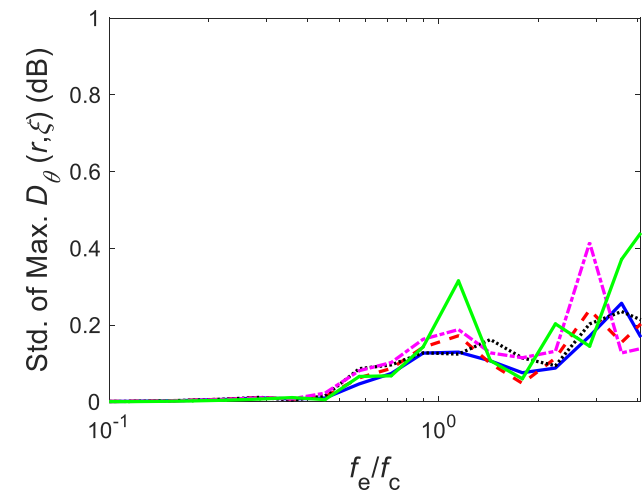

(b)

Figure 15. Variation of averaged angular directivity with frequency for the concrete plate strips of different aspect ratio $(b / a)$ and subjected to 25 incoherent forces across the plate (SSSS, $r / a=10, h=125 \mathrm{~mm}$ ): (a) maximum value of $D_{\theta}(r)$; (b) standard deviation of maximum values of $D_{\theta}(r, \xi)$ with respect to $\xi$ direction

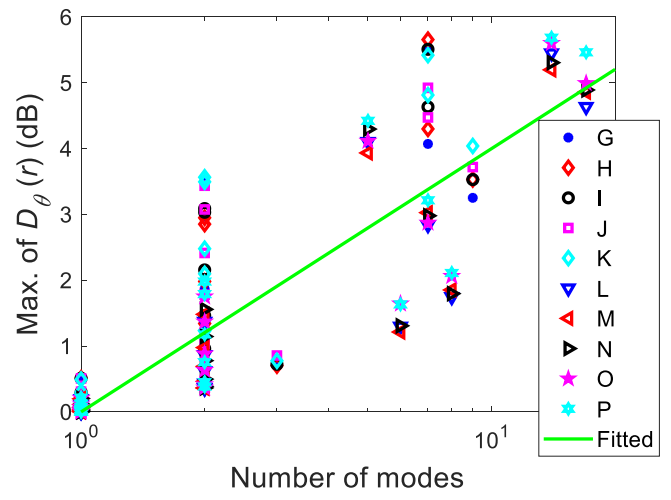

Figure 16. Maximum value of $D_{\theta f}(r)$ against number of modes in each one-third octave band for concrete plate of different sizes and subjected to 25 incoherent forces. 


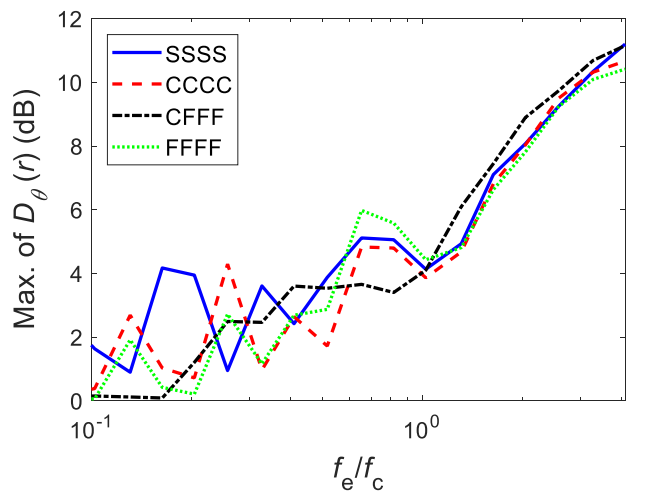

(a)

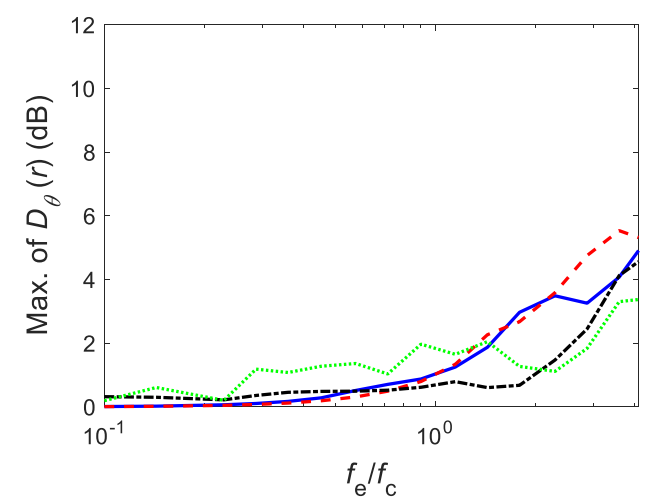

(c)

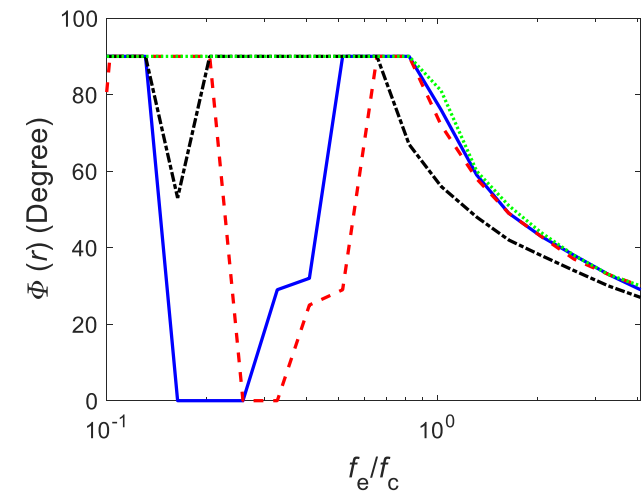

(b)

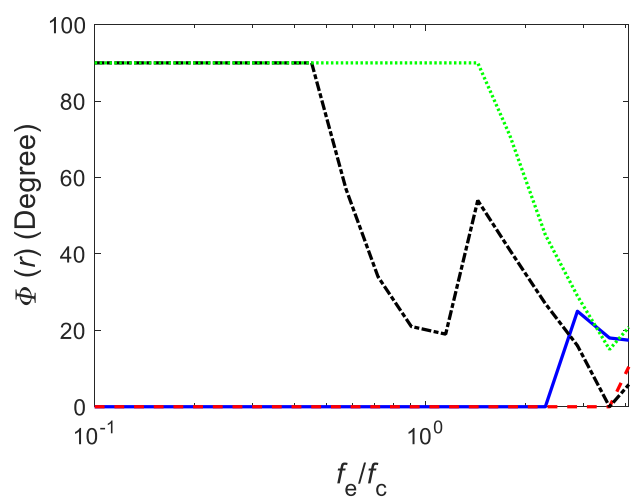

(d)

Figure 17. Variation of averaged angular directivity with frequency for plate strips subjected to 25 incoherent forces across the plate and with various boundary conditions $(r / a=10)$ : (a) Plate $\mathrm{F}$, maximum value of $D_{\theta}(r)$; (b) Plate F, dominant radiation angle; (c) Plate G, maximum value of $D_{\theta}(r)$; (d) Plate G, dominant radiation angle

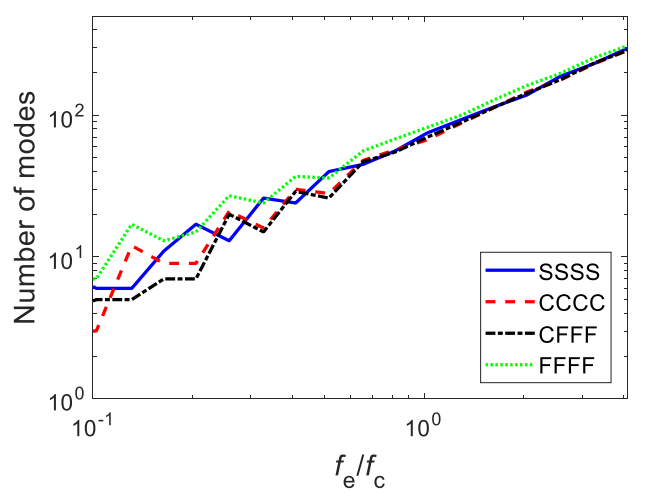

(a)

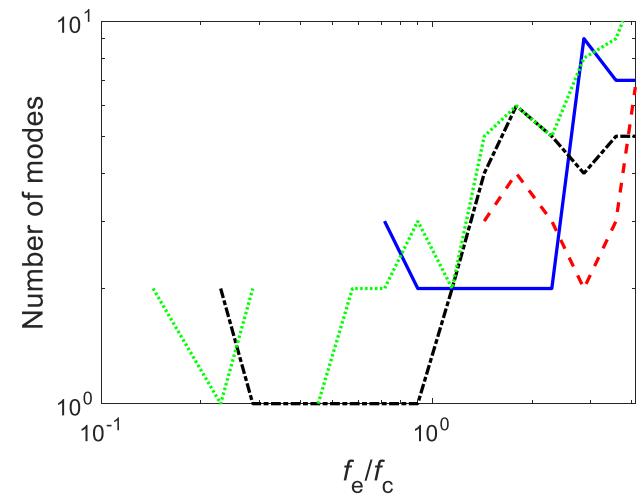

(b)

Figure 18. Number of modes in each $1 / 3$ octave band for plate strips with various boundary conditions: (a) Plate F; (b) Plate G 


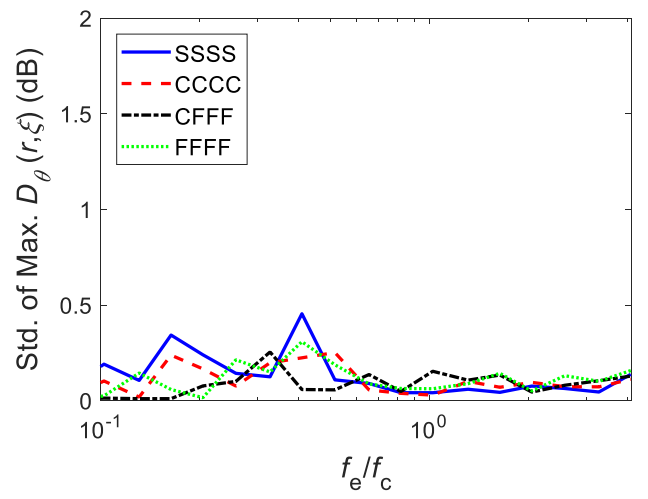

(a)

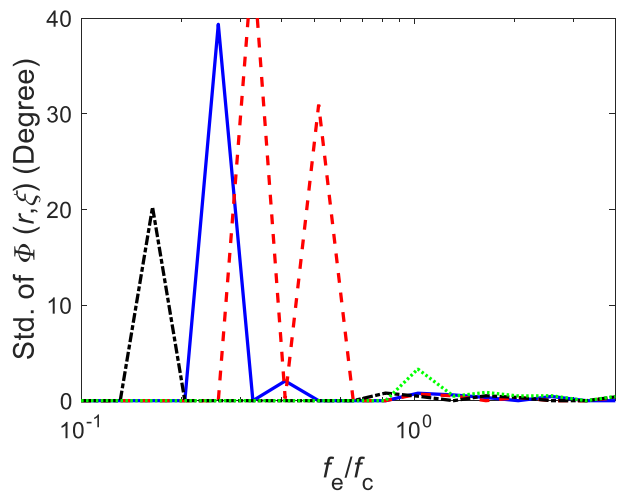

(b)

Figure 19. Variation of averaged angular directivity with frequency for the plate $\mathrm{F}$ with various boundary conditions and subjected to 25 incoherent forces across the plate $(r / a=10)$ : (a) standard deviation of maximum values of $D_{\theta}(r, \xi)$ with respect to $\xi$ direction; (b) standard deviation of the dominant sound radiation angles

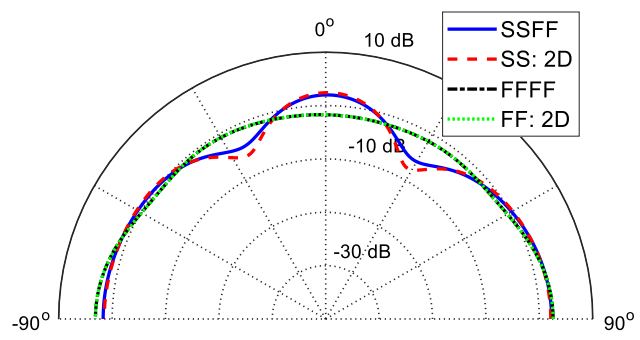

(a)

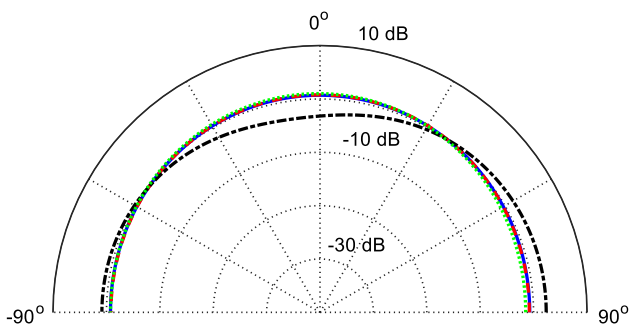

(c)

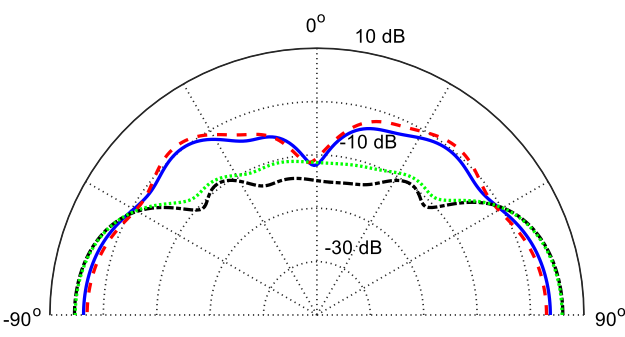

(b)

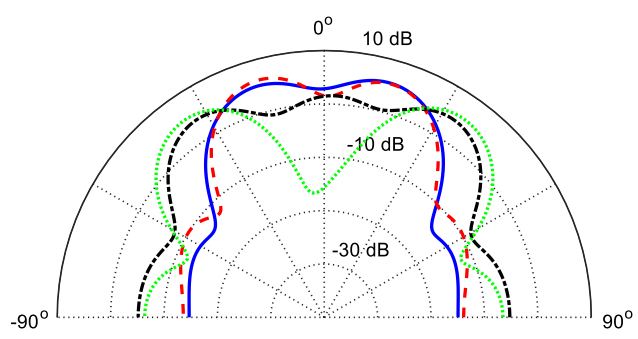

(d)

Figure 20. Averaged angular intensity indices $D_{\theta}(r)$ for the strips vibrating in 3D and 2D modes $\left(x_{f} / a=-\right.$ $0.37, r / a=10$ ): (a) Plate F, $f_{\mathrm{e}}=1000 \mathrm{~Hz}, f_{\mathrm{e}} / f_{\mathrm{c}}=0.82, k a=9.16$; (b) Plate F, $f_{\mathrm{e}}=2000 \mathrm{~Hz}, f_{\mathrm{e}} / f_{\mathrm{c}}=1.64, k a=$ 18.3; (c) Plate G, $f_{\mathrm{e}}=50 \mathrm{~Hz}, f_{\mathrm{e}} / f_{\mathrm{c}}=0.72, k a=1.34$; (d) Plate G, $f_{\mathrm{e}}=315 \mathrm{~Hz}, f_{\mathrm{e}} / f_{\mathrm{c}}=4.52, k a=8.66$ 


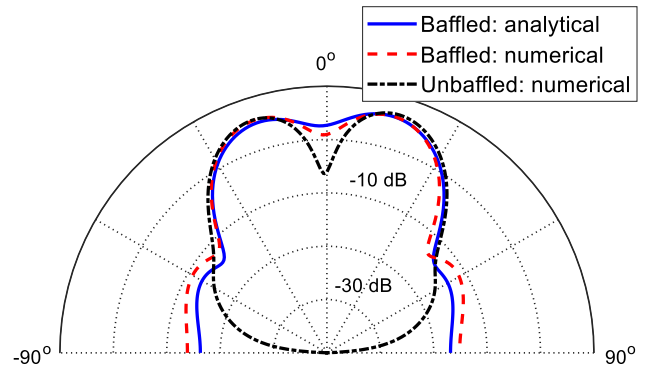

(a)

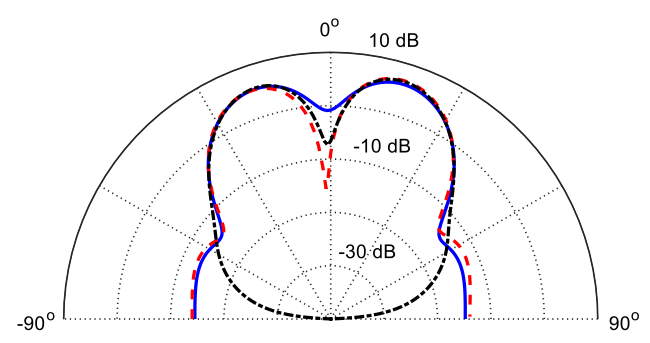

(a)

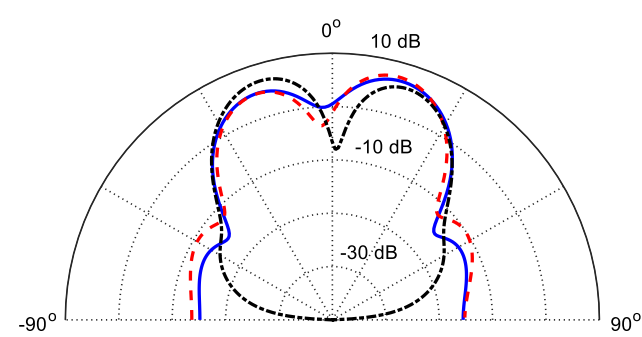

(b)

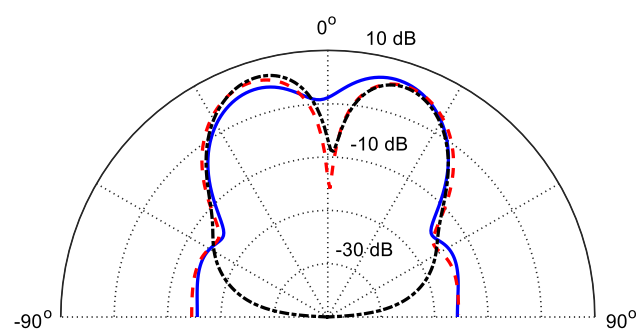

(d)

Figure 21. Angular directivity indices $D_{\theta}(r, \xi)$ for baffled and unbaffled Plate G subjected to 5 incoherent forces along the length direction (SSSS, $r / a=10, x_{f} / a=-0.37, f_{\mathrm{e}}=315 \mathrm{~Hz}, f_{\mathrm{e}} / f_{\mathrm{c}}=4.52, k a=8.66$ ): (a) $\frac{\xi}{b}=$ $\frac{-7}{8}$; (b) $\frac{\xi}{b}=\frac{-5}{8}$; (c) $\frac{\xi}{b}=\frac{-3}{8} ;$ (d) $\frac{\xi}{b}=\frac{-1}{8}$ 